ARTICLE

\title{
Toxic tau oligomer formation blocked by capping of cysteine residues with 1,2-dihydroxybenzene groups
}

Yoshiyuki Soeda ${ }^{1, \dagger}$, Misato Yoshikawa1, Osborne F.X. Almeida ${ }^{2}$, Akio Sumioka $^{1}$, Sumihiro Maeda ${ }^{3}$, Hiroyuki Osada ${ }^{4,5}$, Yasumitsu Kondoh4,5, Akiko Saito ${ }^{6}$, Tomohiro Miyasaka7, Tetsuya Kimura ${ }^{1}$, Masaaki Suzuki ${ }^{8}$, Hiroko Koyama9 ${ }^{9}$ Yuji Yoshiike ${ }^{10}$, Hachiro Sugimoto ${ }^{11}$, Yasuo Ihara 7,12 \& Akihiko Takashima ${ }^{1}$

Neurofibrillary tangles, composed of hyperphosphorylated tau fibrils, are a pathological hallmark of Alzheimer's disease; the neurofibrillary tangle load correlates strongly with clinical progression of the disease. A growing body of evidence indicates that tau oligomer formation precedes the appearance of neurofibrillary tangles and contributes to neuronal loss. Here we show that tau oligomer formation can be inhibited by compounds whose chemical backbone includes 1,2-dihydroxybenzene. Specifically, we demonstrate that 1,2-dihydroxybenzene-containing compounds bind to and cap cysteine residues of tau and prevent its aggregation by hindering interactions between tau molecules. Further, we show that orally administered DL-isoproterenol, an adrenergic receptor agonist whose skeleton includes 1,2-dihydroxybenzene and which penetrates the brain, reduces the levels of detergent-insoluble tau, neuronal loss and reverses neurofibrillary tangle-associated brain dysfunction. Thus, compounds that target the cysteine residues of tau may prove useful in halting the progression of Alzheimer's disease and other tauopathies.

\footnotetext{
${ }^{1}$ Department of Aging Neurobiology, National Center for Geriatrics and Gerontology, Obu, Aichi 474-8511, Japan. ${ }^{2}$ Department of Stress Neurology and Neurogenesis, Max Planck Institute of Psychiatry, Kraepelinstrasse, 2-10, Munich 80804, Germany. ${ }^{3}$ Gladstone Institute of Neurological Disease, University of California, San Francisco, California 94158-2261, USA. ${ }^{4}$ Chemical Biology Research Group, RIKEN Center for Sustainable Resource Science (CSRS), RIKEN, Wako, Saitama 351-0198, Japan. ${ }^{5}$ Antibiotics Laboratory, Advanced Science Institute, RIKEN, Wako, Saitama 351-0198, Japan. ${ }^{6}$ Graduate School of Engineering, Osaka Electro-communication University (OECU), 18-8 Hatsu-cho, Osaka 572-8530, Japan. ${ }^{7}$ Department of Neuropathology, Faculty of Life and Medical Sciences, Doshisha University, Kyotanabe, Kyoto 610-0394, Japan. ${ }^{8}$ Department of Clinical and Experimental Neuroimaging, Center for Development of Advanced Medicine for Dementia, National Center for Geriatrics and Gerontology, Obu, Aichi 474-8511, Japan. ${ }^{9}$ Division of Regeneration and Advanced Medical Science, Gifu University Graduate School of Medicine, Gifu 501-1194, Japan. ${ }^{10}$ Alzheimer's Disease Project Team, National Center for Geriatrics and Gerontology, Obu, Aichi 474-8511, Japan. ${ }^{11}$ Laboratory of Structural Neuropathology, Graduate School of Brain Science, Doshisha University, Kizugawa, Kyoto 619-0225, Japan. ${ }^{12}$ Laboratory of Cognition and Aging, Doshisha University, Kizugawa 619-0225, Japan. † Present address: Study Promotion Strategy Section/Clinical Research Center, Fukushima Medical University, Hikarigaoka 1, Fukushima 960-1295, Japan. Correspondence and requests for materials should be addressed to A.T. (email: kenneth@ncgg.go.jp).
} 
A lzheimer's disease $(\mathrm{AD})$ is a progressive neurodegenerative disease, initially characterized by impaired episodic memory and eventually, severe cognitive decline. Since age is the most important risk factor for $\mathrm{AD}$ development, the present increase in lifespan across all demographics prioritizes the search for ameliorative and preventative treatments for the disease. Currently, cholinesterase inhibitors and N-methyl-Daspartate receptor antagonists are used to treat $\mathrm{AD}$ symptoms with limited success; the current consensus view is that stopping disease progression will require the development of disease-modifying therapies based on defined pathogenic mechanisms ${ }^{1}$.

Deposition of amyloid $\beta$ (A $\beta$ ) peptide in the extracellular space and formation of senile plaques, as well as the intracellular accumulation of tau protein that gives rise to neurofibrillary tangles (NFTs), with contemporaneous neuronal loss are the key pathological hallmarks of $\mathrm{AD}^{2}$. The amyloid hypothesis of $\mathrm{AD}$ posits that $A \beta$ is the primary cause of dementia owing to its ability to induce the formation of NFT and synaptic and neuronal loss in the neocortex ${ }^{3-7}$. Tau tangle formation occurs downstream from $A \beta$ deposition and appears to be essential for the establishment of $\mathrm{AD}$; the latter view is supported by studies showing that tau depletion prevents $A \beta$-induced memory impairment in various lines of human amyloid precursor protein-overexpressing mice ${ }^{8,9}$.

To date, $A \beta$-targeted therapies (for example, immunotherapy) have failed for a variety of reasons, including failure to hamper neurodegeneration and cognitive dysfunction in patients with $\mathrm{AD}^{10,11}$. The focus of $\mathrm{AD}$ drug discovery research has recently shifted towards tau ${ }^{12}$ because, in contrast to $A \beta$ load, tau pathology correlates with the degree of cognitive impairment ${ }^{13,14}$ and neuronal loss ${ }^{15,16}$. Tau is an attractive target because patients with frontotemporal dementia and Parkinsonism-linked to chromosome-17 (FTDP-17) carry a mutation in the tau gene and display NFT and neuronal loss in the absence of $\mathrm{A} \beta$ deposition $^{17-19}$. Moreover, mice overexpressing mutant tau exhibit NFTs, neuronal loss and behavioural abnormalities ${ }^{20-24}$. Several lines of evidence, based on the results of experiments involving expression of the human P301L transgene in mice ${ }^{25,26}$ or the human FTDP17 mutation in fruit flies ${ }^{27}$, link neuronal death to the tau aggregation process, rather than to NFT formation.

Tau protein in NFTs is highly phosphorylayed, reflecting an imbalance in the activities of various kinases and phosphatases ${ }^{28,29}$. Although the role of phosphorylation in the Tau aggregation process is still debated (for example, in vitro evidence suggests Tau phosphorylation inhibits ${ }^{30}$ or has no role on tau aggregation ${ }^{31}$ ), hyperphosphorylated and/or mutated tau is suggested to adopt an alternative structure that promotes interactions between individual tau molecules. For example, our own in vitro experiments have proposed that tau aggregation occurs in a step-wise manner: initially, tau molecules bind to each other, through disulfide binding of their Cys residues ${ }^{32}$, to form soluble tau oligomers ${ }^{32,33}$; in a second step, these oligomers, comprised of $\sim 40$ tau molecules, grow and precipitate as granular tau oligomers with a $\beta$-sheet structure; last, the granular tau oligomers bind to each other and form tau fibrils ${ }^{33}$.

Granular tau oligomers are detectable in the prefrontal cortex at Braak stage I, whereas NFT appear much later (Braak stage $\mathrm{V})^{34}$, indicating that their formation represents a crucial early pathogenic event. Observations that neuronal death is strongly associated with the presence of Sarkosyl-insoluble tau ${ }^{26}$, imply that granular tau oligomers with a $\beta$-sheet structure are a major toxic species of tau and that prevention of their formation could be a promising therapeutic strategy ${ }^{35}$. Following this rationale, we screened a small-molecule library for compounds with the potential to inhibit the formation of granular tau oligomers. We report here that compounds containing 1,2-dihydroxybenzene inhibit granular tau oligomer formation by modifying the Cys residues of tau, thereby reducing Sarkosyl-insoluble tau levels, neuronal death and brain dysfunction in $\mathrm{P} 301 \mathrm{~L}$ tau-transgenic mice.

\section{Results}

Chemical array screening for tau aggregation inhibitors. To find an inhibitor of granular tau oligomer formation, we screened a series of tau-binding compounds, using a small-molecule array consisting of 6,788 compounds in the RIKEN Natural Products Depository (NPDepo). This initial screen led to the identification of 86 compounds displayed the potential to associate with tau. These compounds were subsequently assayed for thioflavin $\mathrm{T}$ (ThT) binding, with the exclusion of false positives using a pelleting assay in which tau was quantified in pellets derived from ultracentrifugation of a mixture of tau aggregates. Three compounds, epinephrine (Fig. 1a), pyrocatechol violet (Fig. 1b) and lobaric acid (Fig. 1c), markedly decreased ThT binding (Fig. 1d-f) and insoluble (aggregated) tau in the pellet fraction (Fig. 1g-i). As the chemical backbones of epinephrine and pyrocathechol violet consist of 1,2-dihydroxybenzene (Fig. 1a,b), we hypothesized that 1,2-dihydroxybenzene endows these compounds with the ability to inhibit tau aggregation.

Inhibition of tau aggregation by 1,2-dihydroxybenzene. Supporting our hypothesis, we observed dose-dependent reductions in heparin-induced ThT fluorescence when cell-free preparations containing recombinant wild-type $2 \mathrm{~N} 4 \mathrm{R}$ tau were exposed to a series of 1,2-dihydroxybenzene-containing compounds. Results are shown for L-3,4-dihydroxyphenylalanine (Fig. 2a), dopamine (Fig. 2b), norepinephrine (Fig. 2c), epinephrine (Fig. 2d) and isoproterenol (ISO; Fig. 2e); adrenochrome, an oxidized form of epinephrine (Fig. 2f), also reduced ThT fluorescence. Octopamine, a compound with a 1-dihydroxybenzene structure, and 3-methoxytyramine, a catechol-O-methyltransferase-mediated metabolite of dopamine, did not alter ThT fluorescence (Fig. 2g,h), verifying the specificity of the effects produced by the compounds with a 1,2-dihydroxybenzene skeleton. This set of data showed that compounds with a 1,2-dihydroxybenzene skeleton can inhibit tau aggregation independently of their oxidation state. Subsequent investigations focused on ISO, a $\beta 1 / 2$ adrenergic receptor agonist that can cross the blood-brain barrier $(\mathrm{BBB})^{36,37}$; ISO is indicated for a number of medical conditions and neither interferes with neurotransmission nor induces major adverse effects. ISO treatment of Neuro2A cells engineered to stably express P301L $\mathrm{tau}^{38}$ results in dose-dependent reductions of SDS-insoluble tau levels in the absence of alterations in the levels of soluble tau (Fig. 3a). Importantly, the inhibitory actions of ISO on tau aggregation were not blocked by pretreatment of P301L tau-expressing Neuro2A cells with propranolol $(1-10 \mu \mathrm{M})$, a competitive antagonist of the $\beta$ adrenergic receptor (Fig. 3b), indicating that the inhibitory action of ISO on tau aggregation is not mediated through $\beta$ adrenergic receptors.

We subsequently made a detailed examination of heparin-induced tau aggregates to gain further insight into the mechanism of action of ISO. Sucrose density gradient centrifugation demonstrated the absence of filamentous tau in ISO-treated $(100 \mu \mathrm{M})$ tau aggregate mixtures (fractions 4-6; Fig. 4a). This analysis also showed that ISO markedly reduced granular tau oligomer levels (fraction 3) while increasing the levels of tau in fractions 1-2 (Fig. 4a). Analysis of heparin-induced tau aggregates by atomic force microscopy 
a

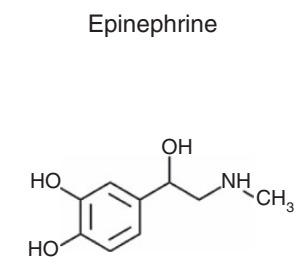

d

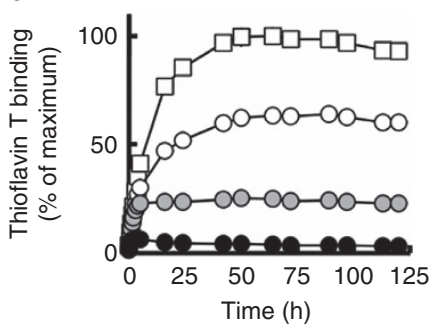

g

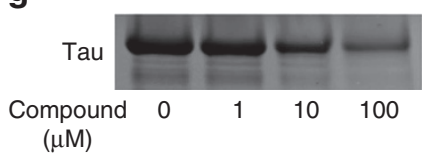

b

Pyrocatechol violet<smiles>O=C1C=CC(=C(c2ccc(O)c(O)c2)c2ccccc2S(=O)(=O)O)C=C1O</smiles>

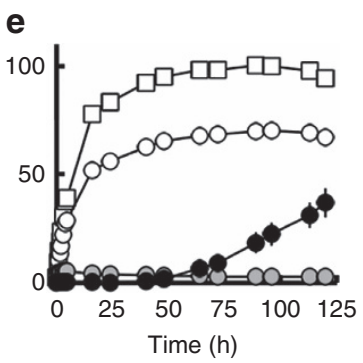

h

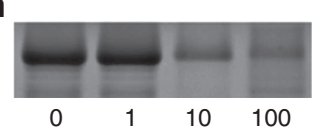

C Lobaric acid

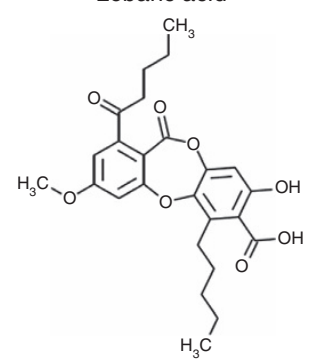

f

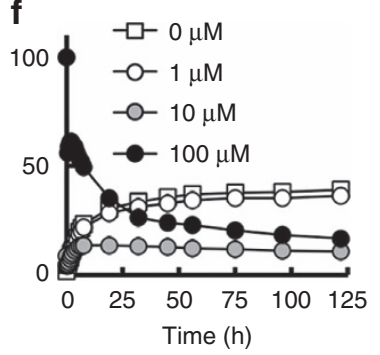

i

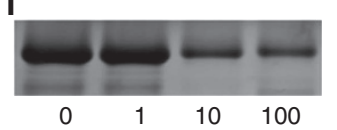

Figure 1 | Identification of tau aggregation inhibitor. Epinephrine $(\mathbf{a}, \mathbf{d}, \mathbf{g})$, pyrocatechol violet $(\mathbf{b}, \mathbf{e}, \mathbf{h})$ and lobaric acid (c,f,i) were screened as tau aggregation inhibitors. Inhibitory effects of tau aggregation were determined by fluorescence of thioflavin $\mathrm{T}(\mathbf{d}, \mathbf{e}, \mathbf{f})$ and pelleting assay $(\mathbf{g}, \mathbf{h}, \mathbf{i})$ of heparin-induced tau polymerization incubated with various concentrations of compounds (1,10 and 100 $\mu \mathrm{M})$. Dimethyl sulfoxide was used as the vehicle. Thioflavin T fluorescence was measured at the indicated time, and results were represented as percentage of maximum thioflavin $T$ fluorescence (d,e,f;mean \pm s.d. of triplicate experiments; $n=3$ ).

(AFM) revealed that ISO treatment leads to a significant reduction of the number of tau filaments and granular tau oligomers and an increase in the number of small tau granules (Fig. 4b). These observations suggest that ISO maintains tau in a soluble form or limits its conversion to small, amorphous granules. This interpretation is consistent with our finding that, under non-reducing conditions, ISO prevents tau molecules from forming soluble oligomers (Fig. 4c).

Inhibitory mechanism of 1,2-dihydroxybenzene. ISO-coated magnetic FG (Functional magnetic) beads (Supplementary Fig. 1) were used to identify the binding site of 1,2-dihydroxybenzene compounds to tau; for this, FG beads were incubated with tau in the presence $(+)$ or absence $(-)$ of ISO before elution with either $1 \mathrm{M} \mathrm{KCl}$ or sample buffer (SB). As shown in Fig. 5a, tau-ISO complexes could be efficiently ut not $\mathrm{KCl}$, indicating that the protein-drug interaction depends on non-ionic bonding. Further, we observed that ISO bound monomeric, dimeric, trimeric and tetrameric forms of tau. Compared with when ISO was not included in the incubation mixture, FG beads bound monomeric tau more strongly (14.1-fold) than dimeric tau (2.2-fold) in the presence of ISO, whereas the amounts of trimeric and tetrameric tau bonding to the FG beads were not influenced by ISO (Fig. 5b). Thus, these results demonstrate that ISO primarily associates with monomeric species of tau.

The site at which ISO binds to tau was further explored by comparing the binding of the drug to wild-type tau and a mutant form of tau that lacks the microtubule-binding region ( $\triangle \mathrm{MTBR}$ ). As tau could only be eluted (recovered) when wild-type tau was used (Fig. 5c), we conclude that ISO binding to tau depends on the presence of MTBR. More precise information regarding the ISO-MTBR site of interaction was obtained by pre-treating FG beads with peptides corresponding to each of the four repeats of tau (R1-R4) before the addition of the drug and subsequent recovery of ISO-tau complexes. Such recovery was only possible from beads that had been pre-treated with vehicle or R1 and R4 peptides (Fig. 5d), indicating that ISO binds to sites localized in the $\mathrm{R} 2$ and/or $\mathrm{R} 3$ regions of tau.

The R2 and R3 regions of tau are characterized by cysteine residues (positions 291 and 322) and hexapeptides (positions 275-280 in $\mathrm{PHF}^{*}$; positions 306-311 in PHF6); these regions are critical for tau fibril formation ${ }^{39-41}$. By testing the ability of ISO to bind to deletion mutants of tau ( $\triangle \mathrm{PHF} 6-$ and $\triangle \mathrm{PHF}^{*}-2 \mathrm{~N} 4 \mathrm{R}$ tau) and in mutant forms of tau in which Cys was substituted by Ala (C291, 322A-2N4R and C322A-2N3R tau), we observed associations between ISO, $\triangle \mathrm{PHF} 6-$ and $\triangle \mathrm{PHF}^{*}-2 \mathrm{~N} 4 \mathrm{R}$ tau, but not C291, 322A-2N4R and C322A-2N3R tau (Fig. 5e); this demonstrated that ISO binds Cys residues in tau. Based on this and other results reported above, we propose that 1,2-dihydroxybenzene non-ionically binds Cys residues in the MTBR and thus inhibits the formation of tau oligomers by occluding intermolecular tau interactions. We next used mass spectrometry to confirm that 1,2-dihydroxybenzene binds Cys residues in tau; for this, a partial Cys-containing peptide of tau, R3 (skvtskcgslgn; molecular weight $(\mathrm{MW})=1,180.3)$, was analysed after incubation with either the vehicle or the 1,2dihydroxybenzene-containing compounds, ISO $(\mathrm{MW}=211.3)$ and pyrocatechol $(\mathrm{MW}=110.1)$. Incubation of the R3' peptide with the vehicle yielded two peaks $(\mathrm{m} / z=1,180.5$ and 2,358.0), corresponding to peptide monomers and dimers (Fig. 6a); incubation with ISO resulted in a distinct peak $(m / z=1,389.7)$, 
a
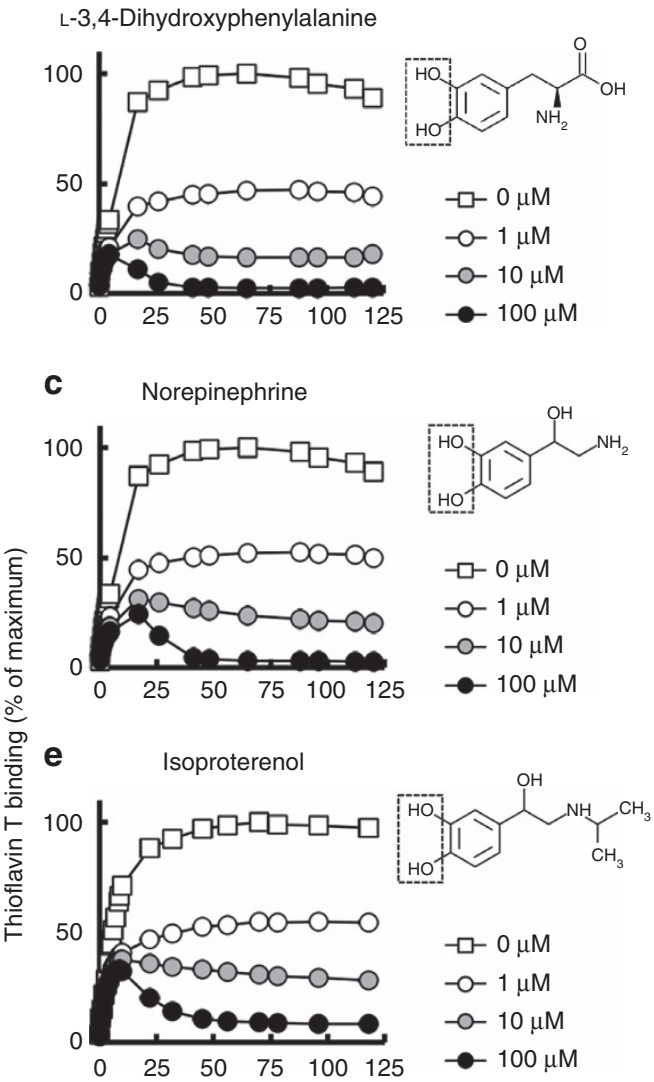

g 3-Methoxytyramine

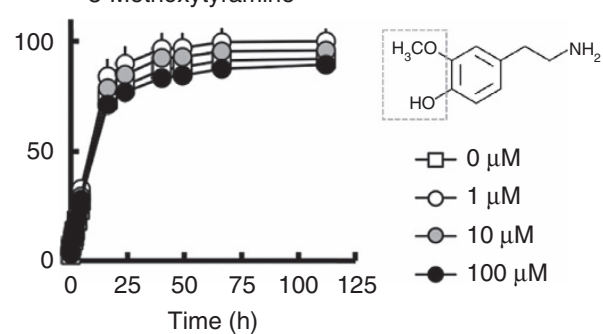

b
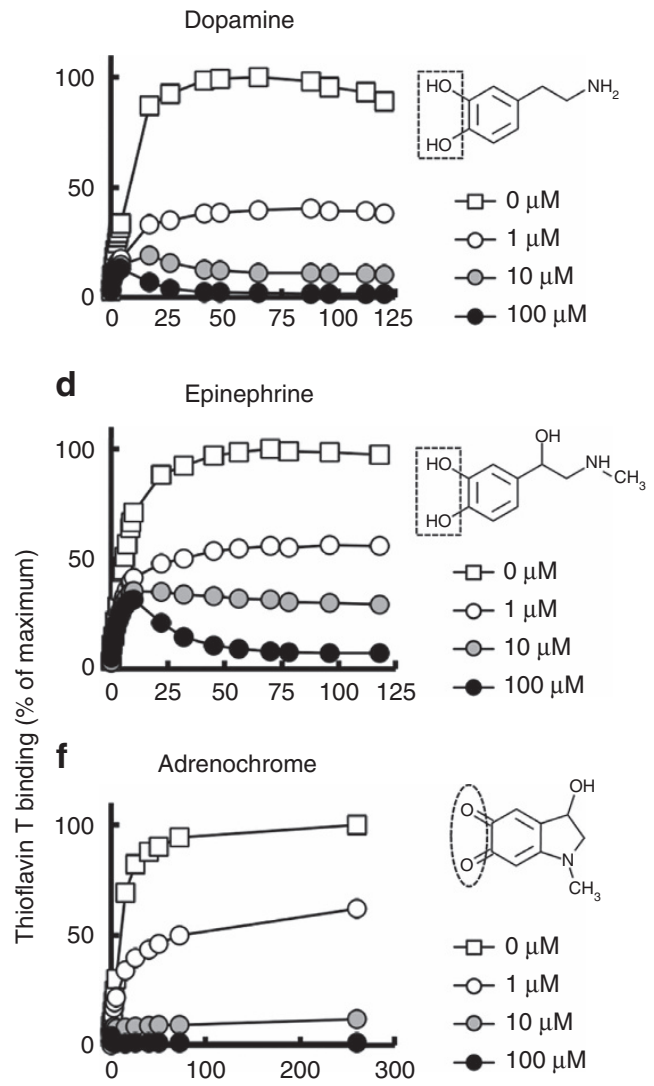

h

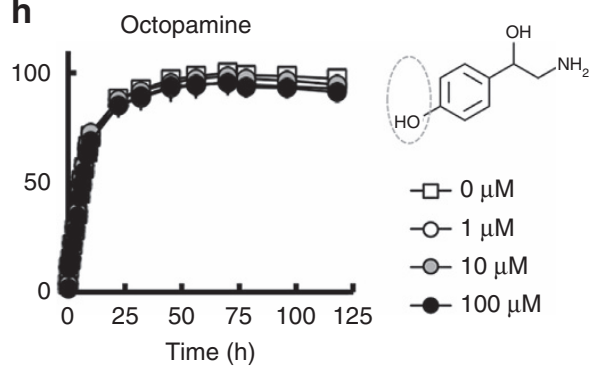

Figure 2 | Inhibitory effects of 1,2-dihydroxybenzene-containing compounds and their derivative compounds on tau aggregation. Thioflavin T-binding activity was dose-dependently inhibited by L-3,4-dihydroxyphenylalanine (a), dopamine (b), norepinephrine (c), epinephrine (d) and isoproterenol (e) (1,2-dihydroxybenezene; black dotted square), and adrenochrome (f) (o-quinone; black dotted circle), but not by octopamine (h) (1-hydroxybenzene; grey dotted circle) or 3-methoxythylamine (g) (1-hydroxy-2-methoxybenzene; grey dotted square). Results are shown as percentage of maximum fluorescence (mean \pm s.d. of triplicate experiments; $n=3$ ).

as well as peaks corresponding to the R3' peptide monomers and dimers (Fig. $6 \mathrm{~b}$ and Supplementary Table 1). Peak shifts $(m / z=1,180.6 \rightarrow 1,288.5)$ were also detected after incubation with pyrocatechol (Fig. 6c and Supplementary Table 1), but not with octopamine, a 1-hydroxybenzene containing compound (Fig. 6d). No peak shifts were observed when a mutant Cys $\rightarrow$ Ala R3' peptide (skvtskagslgn; $\mathrm{MW}=1,148.2$ ) was incubated with the vehicle (Fig. 6e), ISO (Fig. 6f), pyrocatechol (Fig. 6g) or octopamine (Fig. 6h). Together, these analyses confirmed that ISO could block tau aggregation through interaction of its 1,2-dihydroxybenzene group with Cys residues in tau.

Effects of ISO in an animal model of tauopathy. To investigate the efficacy of ISO in inhibiting tau aggregation and subsequent neuronal loss in a relevant animal model of tauopathy, we used a transgenic mouse line that expresses a highly aggregating form of tau, encoded by the human P301L tau gene. Studies were performed in aged mice (17-18 months) as the amount of Sarkosylinsoluble tau aggregate in the brain increases with age ${ }^{26}$. Animals (non-transgenic controls and P301L tau transgenic mice) received DL-ISO in their chow at dose of $1.5 \mathrm{mg} \mathrm{g}^{-1}$ chow; food intake and body weight did not change over the duration (3 months) of treatment (Supplementary Fig. 5). The oral DL-ISO regimen did not alter TBS-soluble levels of tau, but significantly reduced the load of Sarkosyl-insoluble tau in the cortex $(26.1 \pm 14.5 \% ; n=12)$, $P=0.01482$ (unpaired Welch's $t$-test), Fig. 7a) and hippocampus $(36.7 \pm 28.3 \% \quad(n=8), \quad P=0.03174$ (unpaired Student's $t$-test) Fig. 7b), as compared with mice fed the control diet. Appropriate drug dosage was established in pilot studies in wild-type mice; following administration of ISO at $1.5 \mathrm{mg} \mathrm{g}^{-1}$ chow for 2 weeks, their blood and brain levels of ISO were 257 and $40 \mathrm{nM}$, 
a

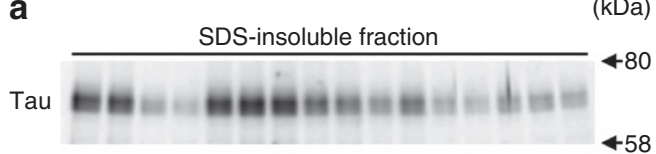

RIPA-soluble fraction
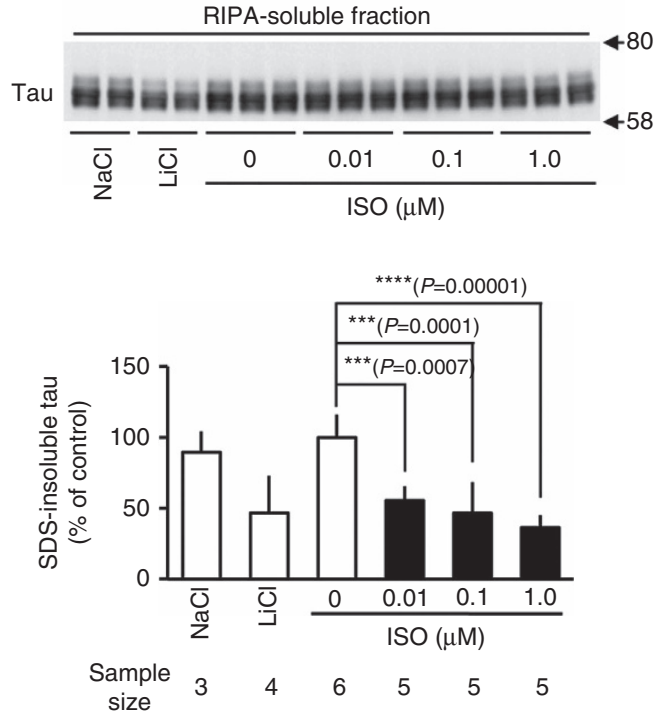

b
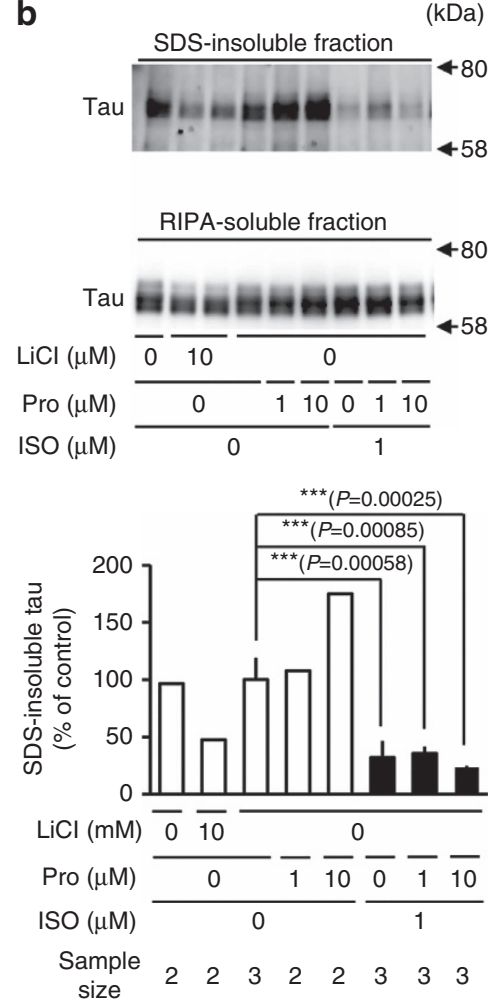

Figure 3 | Isoproterenol inhibits tau aggregation independent of $\boldsymbol{\beta}$ adrenergic stimulation in cultured cells. Neuro-2a cells expressing human $2 \mathrm{~N} 4 \mathrm{R}$ tau (P301L) were treated with 0 (milliQ water), $0.01,0.1$ and $1 \mu \mathrm{M}$ isoproterenol, $10 \mathrm{mM}$ lithium chloride as a positive control as an inhibitor of glycogen synthase kinase and $10 \mathrm{mM}$ sodium chloride as a negative control for $48 \mathrm{~h}$. SDS-insoluble (a, upper part of panel) and RIPA-soluble fractions (a, middle part of panel) were obtained from the cell homogenates and were subjected to immunoblot analysis with JM antibody that recognized total tau. Immunoreactivity was quantified (a, lower part of panel), and levels of SDS-insoluble tau were normalized by corresponding RIPA-soluble tau. Results are represented as percentage of control (mean \pm s.d. of 3-6 experiments; $10 \mathrm{mM}$ sodium chloride $(n=3), 10 \mathrm{mM}$ lithium chloride $(n=4)$, vehicle $(n=6)$, $0.01 \mu \mathrm{M}$ isoproterenol $(n=5), 0.1 \mu \mathrm{M}$ isoproterenol $(n=5), 1 \mu \mathrm{M}$ isoproterenol $(n=5))$. ${ }^{\star \star \star} P<0.001$; ${ }^{\star \star \star \star} P<0.0001$ (one-way analysis of variance (ANOVA), with Tukey's multiple comparisons test). (b) Neuro-2a cells expressing human $2 \mathrm{~N} 4 \mathrm{R}$ tau (P301L) were pretreated with 0,1 and $10 \mu \mathrm{M}$ propranolol $30 \mathrm{~min}$ before treatment with $\mathrm{O}$ (milliQ water), $1 \mu \mathrm{M}$ isoproterenol, $10 \mathrm{mM}$ lithium chloride and $10 \mathrm{mM}$ sodium chloride for $48 \mathrm{~h}$. SDS-insoluble (b, upper part of panel) and RIPA-soluble (b, middle part of panel) fractions were obtained and subjected to immunoblot using JM antibody.

Immunoreactivity was quantified (b, lower part of panel) and levels of SDS-insoluble tau were normalized to corresponding RIPA-soluble tau. Results are represented as percentage of control (mean \pm s.d. of 2-3 experiments; $10 \mathrm{mM}$ sodium chloride $(n=2), 10 \mathrm{mM}$ lithium chloride $(n=2)$, vehicle $(n=3)$, $1 \mu \mathrm{M}$ propranolol ( $n=2), 10 \mu \mathrm{M}$ propranolol $(n=2), 1 \mu \mathrm{M}$ isoproterenol $(n=3), 1 \mu \mathrm{M}$ propranolol/ $1 \mu \mathrm{M}$ isoproterenol $(n=3), 10 \mu \mathrm{M}$ propranolol/1 $\mu \mathrm{M}$ isoproterenol $(n=3)$ ). ${ }^{\star \star \star} P<0.001$ (one-way ANOVA, with Tukey's multiple comparisons test). ISO, isoproterenol; Pro, propranolol.

respectively. Potential mediation of the actions of ISO by $\beta 1 / 2$ adrenergic receptors was precluded because similar reductions of Sarkosyl-insoluble tau were observed in both cerebral cortex (Fig. 7c) and hippocampus (Fig. 7d) when P301L tau-transgenic mice were fed for 3 months with the D-isomer of the drug (1.5 $\mathrm{mg} \mathrm{g}^{-1}$ chow), which lacks adrenergic activity (cf. Fig. 7a,b, respectively). In addition, administration of the inactive isomer of ISO for 2 months resulted in dose-dependent reductions of Sarkosyl-insoluble tau (Fig. 7e). The latter two observations indicate that the ability of D/DL-ISO to inhibit tau aggregation are dose- and time-dependent.

Our previous observations that aged P301L tau-transgenic mice display neuronal loss in the entorhinal cortex, temporal lobe and amygdala $^{26}$ were reproduced in the present study (Fig. $7 \mathrm{f}-\mathrm{h}$ ). Having previously attributed these effects to the increased levels of insoluble Sarkosyl-insoluble (aggregated) tau in the brains of aged P301L tau-transgenic mice 26 , we hypothesized that ISO would promote neuronal survival by reducing tau aggregation. Our hypothesis was supported by the results depicted in Fig. $7 f-h$, where ISO ( $1.5 \mathrm{mgg}^{-1}$ chow for 3 months) is seen to prevent agerelated reductions in neuron numbers in the entorhinal cortex, temporal lobe and basolateral amygdala of aged P301L tau- transgenic mice; the drug did not influence neuron numbers in any of these areas in non-transgenic mice.

On the basis of above findings, we suggest that inhibition of tau aggregation via ISO-capping of Cys residues of tau prevents the aggregation of toxic tau and therefore, neuronal loss. Such a mechanism is supported by the observation that D-ISO $\left(15 \mathrm{mgg}^{-1}\right.$ chow) reduces Sarkosyl-insoluble tau and blocks neuronal loss in the hippocampus of PS19 mice (a model of tauopathy which also displays severe hippocampal neuronal $\operatorname{loss}^{20}$ ); the latter conclusion is supported by the magnetic resonance imaging data presented in Supplementary Fig. 2. Although the neuronal loss observed in P301L tau-transgenic mice was not accompanied by significantly impaired spatial memory in the Morris water maze test ${ }^{26}$, they displayed significantly reduced locomotor activity during the first minute of placement in an open field arena (Fig. 7i). On the other hand, their locomotor activity over $30 \mathrm{~min}$ in the open field arena did not differ from that observed in non-transgenic mice (Fig. 7j); these observations suggest that P301L tau-transgenic mice exhibit emotional disturbance without any change in overall motor activity. Interestingly, ISO alleviated the emotional disturbance in aged P301L tau-transgenic mice (Fig. 7i). 

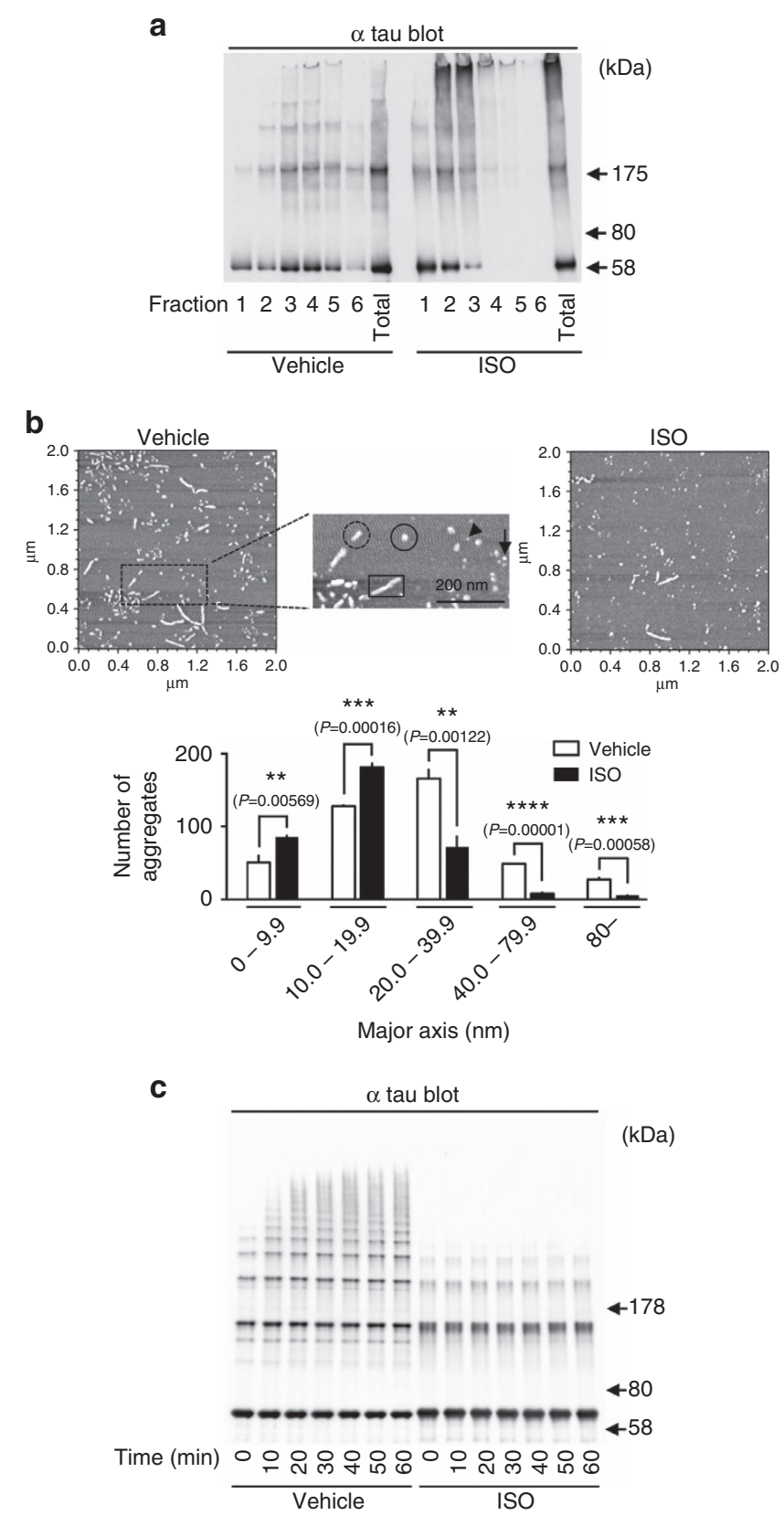

Figure 4 | Inhibition of tau oligomer formation by isoproterenol. Heparin-induced tau aggregation mixture ( $120 \mathrm{~h}$ incubation) was subjected to sucrose density gradient centrifugation (a) and AFM observation (b). Tau aggregation mixture was separated into six fractions, and levels of tau in each fraction were analysed by western blot using tau 5 antibody that recognized total tau. In AFM observation, three images were obtained from different areas $\left(2 \times 2 \mu \mathrm{m}^{2}\right)$ of the mica and the number and size of tau aggregates was quantitated using the image analysis programme, Matlab. Sizes of tau aggregates are $0-9.9 \mathrm{~nm}$ (arrowhead), 10.0-19.9 nm (arrow), 20.0-39.9 nm (black solid circle), 40.0-79.9 nm (black dotted circle) and not less than $80 \mathrm{~nm}$ (black solid square). Results represented as mean \pm s.d. of three experiments $\left(n=3\right.$; b, lower panel). ${ }^{\star \star} P<0.01 ;{ }^{\star \star \star} P<0.001 ;{ }^{\star \star \star \star} P<0.0001$ (unpaired Student's $t$-test). In first $60 \mathrm{~min}$ of heparin-induced tau aggregation, higher order tau oligomer was formed in soluble fraction with longer incubation time (c, vehicle), but growth of tau oligomer was not seen in the presence of isoproterenol (c, ISO). Tau oligomer in soluble fraction was detected by non-reducing condition using tau 5 antibody.

\section{Discussion}

Growing evidence for the key role of aggregates of tau protein in $\mathrm{AD}$ and FTDP-17 has spurred efforts to explore pharmacological means to maintain tau in its soluble form and thus, to prevent its aggregation.

ISO was identified as a potential inhibitor of tau aggregation after screening a library of small molecules. Detailed analysis revealed that the drug acts by binding Cys residues in tau that prevent tau oligomerization and formation of insoluble tau aggregates. This property of ISO is attributed to the two phenolic hydroxyl groups in the 1,2-dihydroxybenzene backbone, as other compounds sharing this structure (L-3,4-dihydroxyphenylalanine, dopamine, norepinephrine, epinephrine, and ISO), as well as 1,2-dihydroxybenzene itself, also inhibit tau aggregation, albeit to lesser extents and/or ability to easily traverse the BBB. The prerequisite role of the 1,2-dihydroxybenzene backbone structure is illustrated by the inability of octopamine and 3-methoxytyramine or 3-methoxy-4-hydroxyphenethylamine, whose chemical backbones include 1-hydroxybenzene, to inhibit tau aggregation. Given that ISO is subject to oxidation, our finding that o-quinone (adrenochrome) inhibit tau aggregation as effectively as epinephrine (its non-oxidized parent molecule) is important because it demonstrates that the inhibitory actions of 1,2-dihydroxybenzene-containing compounds on tau aggregation do not depend on their oxidative status. Interestingly, previous work demonstrated nucleophilic covalent attachment of the $o$-quinone in 1,2-dihydroxybenzene compounds to the sulfate group in $\mathrm{Cys}^{42,43}$; consistent with this finding. It would therefore appear that oxidized 1,2-dihydroxybenzene-containing compounds and their o-quinone forms bind covalently to the sulfate group of Cys; this view is plausible because we found that the ISO-bound tau bond cannot be broken eluted by $1 \mathrm{M} \mathrm{KCl}$. The site of chemical reaction between Cys residue of tau and $o$-quinone would be regulated by steric and electronic interactions of these two compounds. Further, MS analysis revealed that complexes comprised of a partial tau R3' peptide and ISO include two phenolic hydroxyl groups, but not $o$-quinone. As shown in the schema in Supplementary Fig. 3, quinone binding to the sulfate group of Cys in tau will be followed by electron transfer to quinone and its back-conversion into ISO (personal communication, M.S. and H.K.) ${ }^{44}$. Oxidation during tau aggregation leads to increased disulfide bonding between Cys residues in tau ${ }^{45,46}$; however, substitution of Cys to Ala in tau (C291, 322A-2N4R tau) can induce tau aggregation, suggesting that capping of Cys residues of tau by 1,2-dihydroxybenzene precludes the formation of toxic tau aggregates by hindering disulfide bonding and formation of tau oligomers (Supplementary Fig. 4a,b). The aggregation of wild-type tau was significantly attenuated by both 10 and $100 \mu \mathrm{M}$ of ISO, whereas that of C291, $322 \mathrm{~A}-2 \mathrm{~N} 4 \mathrm{R}$ tau was only inhibited by the higher dose of ISO (Supplementary Fig. 4c). Besides showing that ISO binds the Cys residue of tau, this observation indicates that ISO also affects PHF6((306) VQIVYK(311) $)^{41,47}$ binding, and thus increasing the propensity to form $\beta$-sheets and inhibiting the formation of seeds for the aggregation of toxic tau.

It deserves mention that, although NFTs themselves do not exert toxic actions ${ }^{25}$, the degree of dementia correlates well with NFT number and the extent of neuronal loss ${ }^{13,14}$. Accordingly, the irreversible brain dysfunction observed in dementia may be ascribed to the neuronal loss that follows the aggregation of toxic tau. Support for this view comes from our finding that ISO reverses emotional disturbances associated with the expression of P301L tau (Fig. 7i) and stimulates neural activity in the prelimbic frontal cortex (Supplementary Fig. 6a); the latter is accompanied by contemporaneous reductions in the levels of Sarkosylinsoluble tau in the prelimbic cortex (Supplementary Fig. 6b). 
a

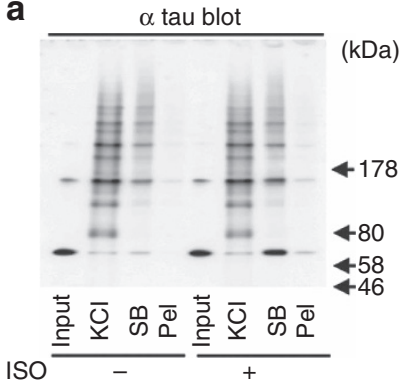

C

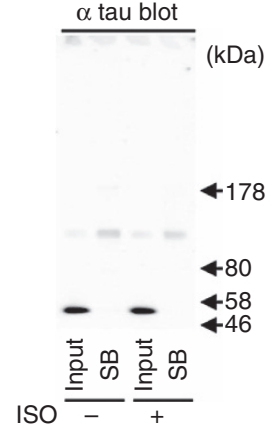

b

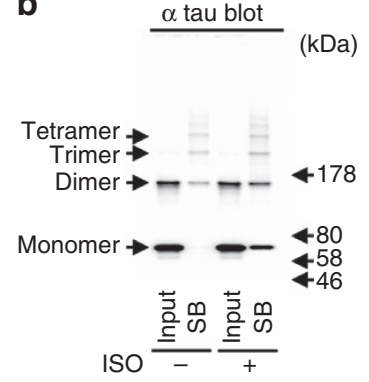

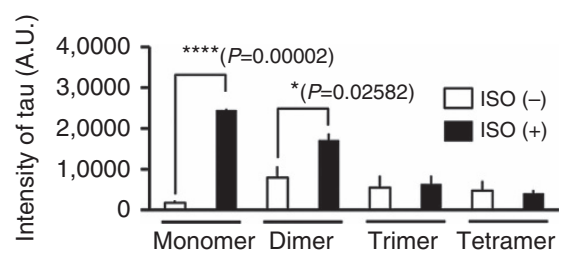

d

$\alpha$ tau blot

$\alpha$ tau blot

(kDa)

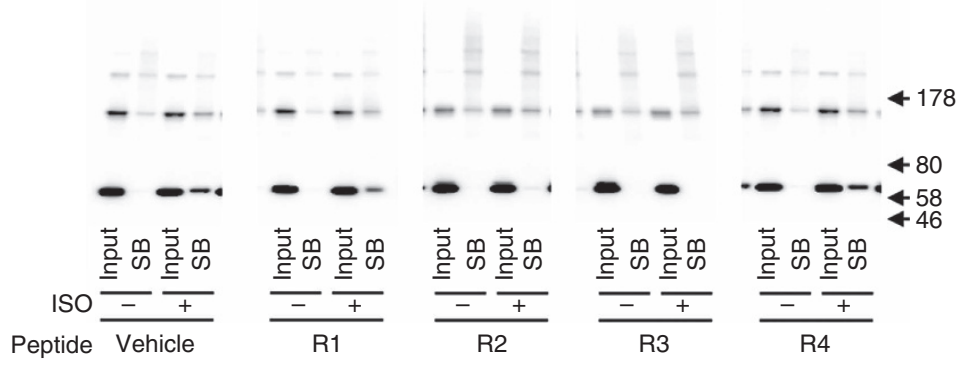

e

$\alpha$ tau blot

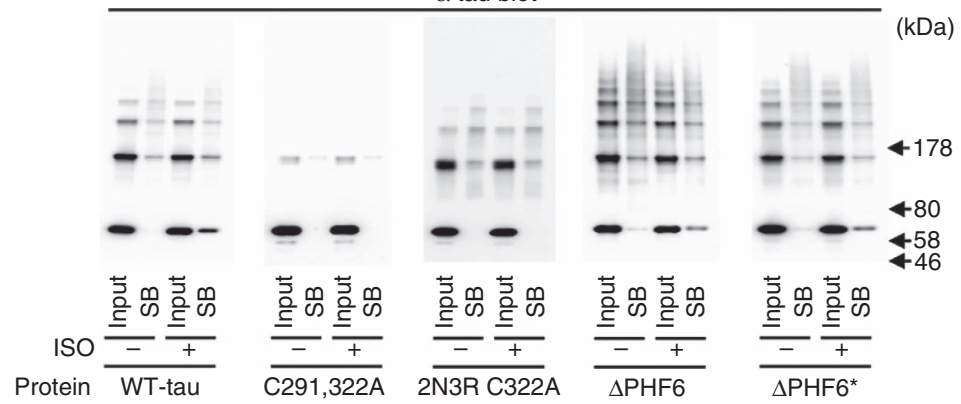

Figure 5 | Identification of isoproterenol (ISO)-binding site on tau. ISO and tau binding were performed according to the protocol described in Supplementary Fig. 1 and ISO-bound tau was recovered in KCI, SB and Pel. Each fraction was subjected to immunoblot analysis with tau5 antibody (a). In SB fraction, tau monomer and oligomer were detected from an eluate of FG beads with ISO (ISO $(+)$ ) and without ISO (ISO ( - ); $\boldsymbol{b}$, left part of panel). Levels of tau monomer and oligomer were quantified (b, right part of panel) and shown as mean \pm s.d. (triplicate experiments; $n=3$ ). ${ }^{\star} P<0.05 ;{ }^{\star \star \star \star} P<0.0001$ (unpaired Student's $t$-test). FG beads with ISO (ISO $(+)$ ) and without ISO (ISO $(-)$ ) were reacted with $\Delta$ MTBR-tau. Tau was not recovered in SB fraction (c). Pretreated ISO with R1 (tapvpmpdlknvkskigstenlkhqpgggk), R2 (vqiinkkldlsnvqskcgskdnikhvpgggs), R3 (vqivykpvdlskvtskcgslgnihhkpgggq) and R4 (vevksekldfkdrvqskigsldnithvpgggn) peptides was incubated with wild-type (WT) 2N4R tau. Pretreated ISO with R2 and R3 could not bind to tau, but R1 and R4 pretreatment could bind (d). C291, 322A-2N4R tau and C322A-2N3R tau did not show tau band as well as tau incubating beads without ISO, ISO ( + ), but WT 2N4R tau, $\triangle$ PHF6-tau and $\triangle$ PHF6 -tau showed tau band as ISO-bound tau (e). MTBR, microtubule-binding domain repeat.

In summary, we have demonstrated that ISO, a drug that penetrates the $\mathrm{BBB}$, efficiently inhibits the formation of Sarkosyl-insoluble tau in a mouse line carrying an aggressive mutant form of tau (P301L); the P301L mutation is responsible for FTDP-17, and mice expressing it are very prone to tau aggregation and neuronal death. Our results point to the potential of 1,2-dihydroxybenzene-based compounds to prevent and delay tauopathies such as FTDP-17 and AD by preventing neuronal dysfunction and loss restoring behavioural homeostasis. Using a variety of analytical approaches, we propose that the therapeutic efficacy of 1,2-dihydroxybenzene-containing compounds hinges on their ability to hinder intermolecular interactions between tau molecules by binding to the Cys residues of tau. No untowards reactions to chronic (3 month) ISO exposure were observed in this study; further, oral administration of the pharmaceutical PROTERENOL $S\left(0.25 \mathrm{mg} \mathrm{kg}^{-1}\right)$ to cynomologus monkeys for 8 weeks was not accompanied by adverse effects (unpublished observations). Nevertheless, the caveat that toxicity might result from covalent modification of Cys residues in other proteins must be considered during subsequent development of ISO-related compounds for therapeutic use. On the other hand, it should be noted that ISO is commonly prescribed for conditions such as asthma and bradycardia, with the therapeutic dosages in humans being almost fivefold those that we found to be effective for disrupting tau aggregation in mice ${ }^{48}$. The present work suggests a promising novel therapeutic target in the management of tauopathies; its screening strategy and description of a series of compounds that display nucleophilie for Cys residues in $\mathrm{tau}^{49}$, provide an important lead in this respect.

\section{Methods}

Materials. Six-thousand seven-hundred eighty-eight small molecular compounds were obtained from the RIKEN Natural Products Depository (NPDepo) and dissolved in dimethyl sulfoxide to obtain a stock solution $\left(10 \mathrm{mM}\right.$ or $\left.10 \mathrm{mg} \mathrm{ml}^{-1}\right)$. The polyclonal anti-total tau antibody (JM) was prepared as previously described $^{50}$. The monoclonal anti-total tau antibody (tau5) was purchased from 
a

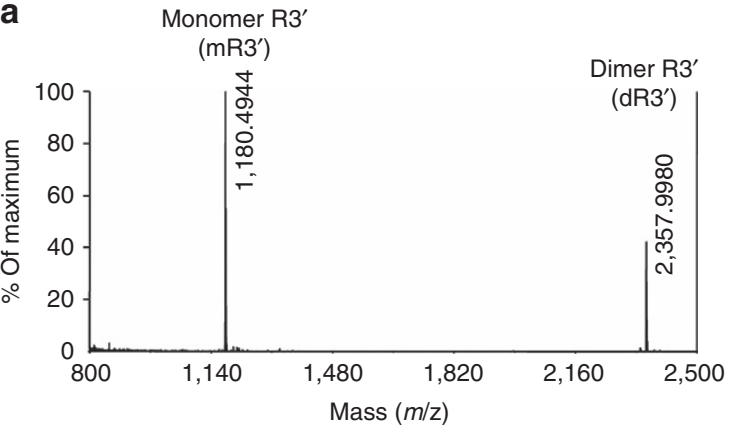

b

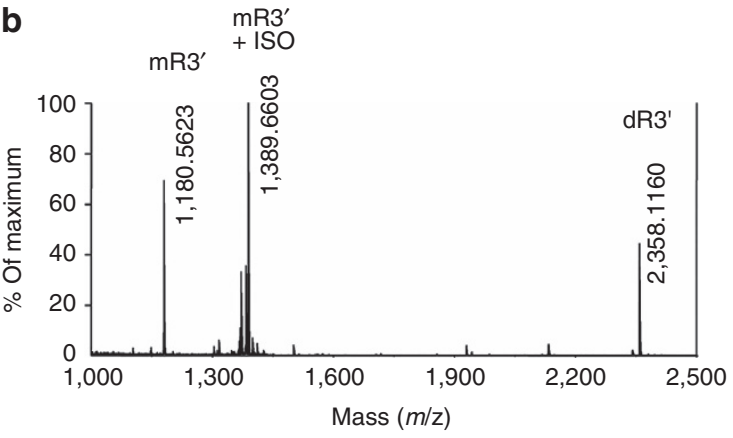

C

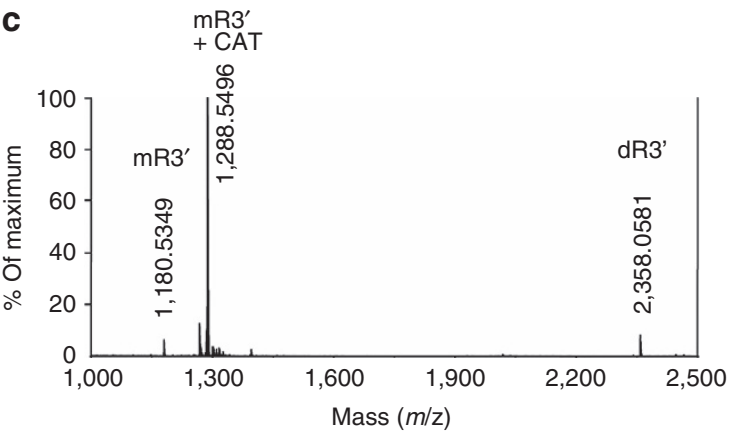

d

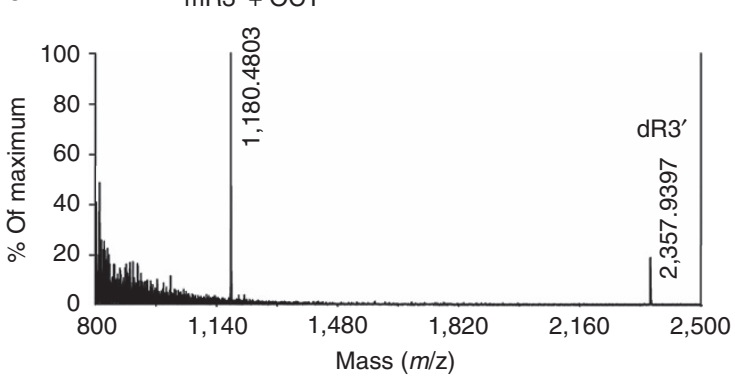

e Monomer mutant $\mathrm{C} \rightarrow \mathrm{A} \mathrm{R} 3^{\prime}$

(mMutant $\left.\mathrm{C} \rightarrow \mathrm{A} \mathrm{R} 3^{\prime}\right)$

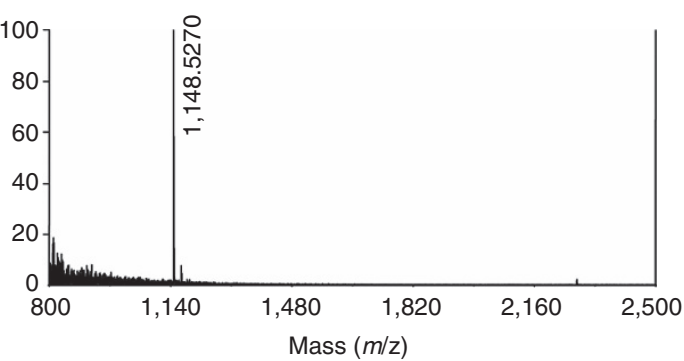

f

mMutant $\mathrm{C} \rightarrow \mathrm{A} \mathrm{R} 3^{\prime}+\mathrm{ISO}$

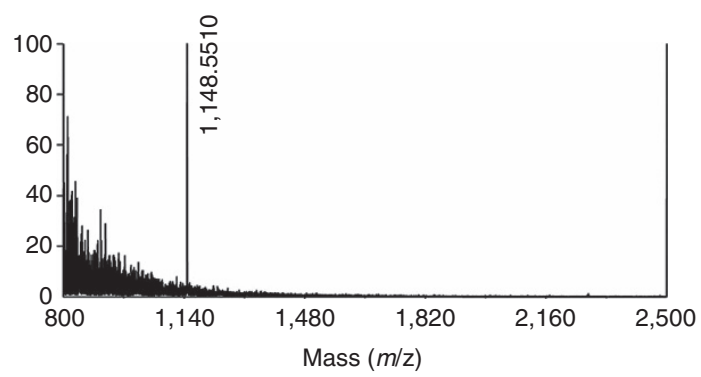

g

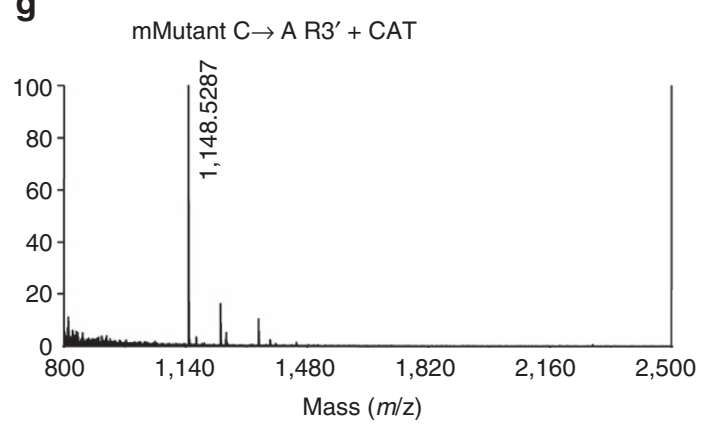

h mMutant $\mathrm{C} \rightarrow \mathrm{AR} 3^{\prime}+\mathrm{OCT}$

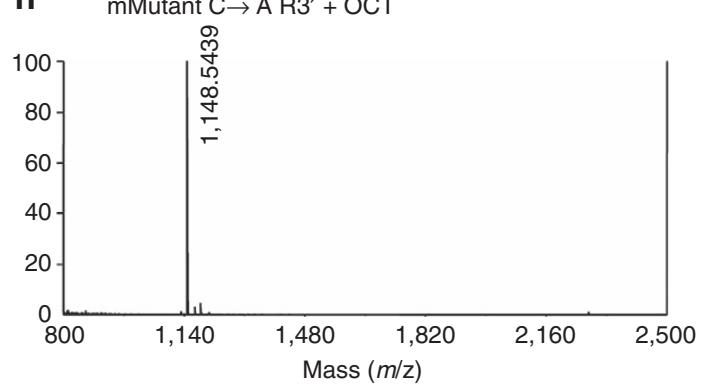

Figure 6 | Binding of 1,2-dihydroxybenzene-containing compounds with Cys residue of tau by mass spectrometry analysis. Vehicle (milliQ water; a) and 1-hydroxybenzene-containing octopamine (OCT; $M W=153.2$; d) were used as negative controls. Compounds containing 1,2-dihydroxybenzene isoproterenol (ISO; $\mathrm{MW}=211.3 ; \mathbf{b})$ and pyrocatechol (CAT; $\mathrm{MW}=110.1 ; \mathbf{c}$ ) incubated with tau R3 partial peptide, R3' ( ISO/R3' and CAT/R3 signal in mass spectrum (b,c). Mutant C $\rightarrow$ A R3' peptide (skvtskagslgn; $M W=1,148.2 ; \mathbf{e}-\mathbf{h}$ ) incubated with vehicle (e), ISO (f), CAT $(\mathbf{g})$ and OCT (h) only showed mutant R3' signal. Data are normalized to the each maximum mass spectrometry signal.

Invitrogen. All other reagents were of analytical grade and purchased from Nacalai Tesque Inc., Sigma-Aldrich Corp. and WAKO Pure Chemical Industries.

Chemical array screening. The method is based on identification of associations between a protein of interest with a library of candidate chemicals. In this study, the array consisted of 6,788 small-molecule compounds (RIKEN Natural Products Depository, NPDepo) immobilized under ultraviolet radiation on to photo-affinity linker-coated glass slides; the assay was performed using a previously reported method $^{51-53}$ with slight modifications. Briefly, after blocking with $1 \%$ skimmed milk in $10 \mathrm{mM}$ HEPES/100 $\mathrm{mM} \mathrm{NaCl}$ for $1 \mathrm{~h}$ at room temperature, the slides were treated with recombinant tau protein in $10 \mathrm{mM} \mathrm{HEPES} / 100 \mathrm{mM} \mathrm{NaCl} / 0.05 \%$

Tween for $16 \mathrm{~h}$ at $4{ }^{\circ} \mathrm{C}$. The slides were then probed with anti-tau antibody (JM) for $4 \mathrm{~h}$, followed by incubation with a Alexa 633-labelled secondary antibody for $1 \mathrm{~h}$ at room temperature. Signal detection was performed with a GenePix 4100A microarray scanner (Molecular Devices), equipped with a 635-nm laser and 655-695 nm band-pass emission filter. When Fraction 1 (soluble tau) was used as bait, 86 out of 6,788 compounds showed potential association with tau. In contrast, none of the compounds tested showed associations either Fraction 3 (granular tau oligomer) or Fraction 6 (fibrilar tau) 

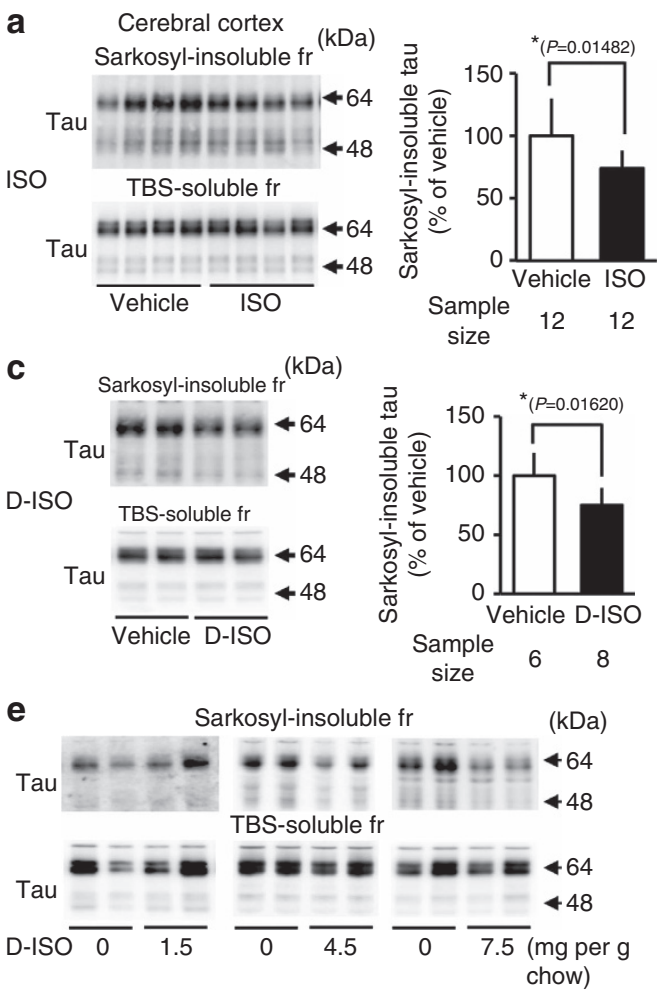
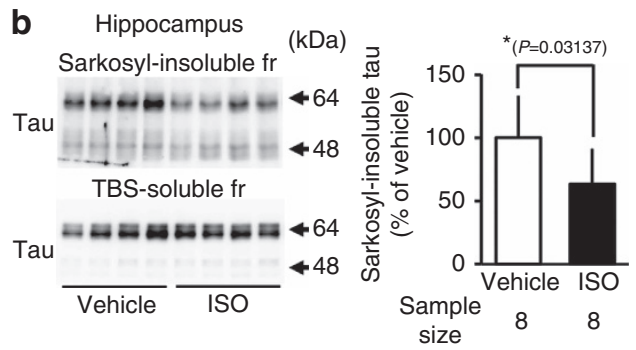

d
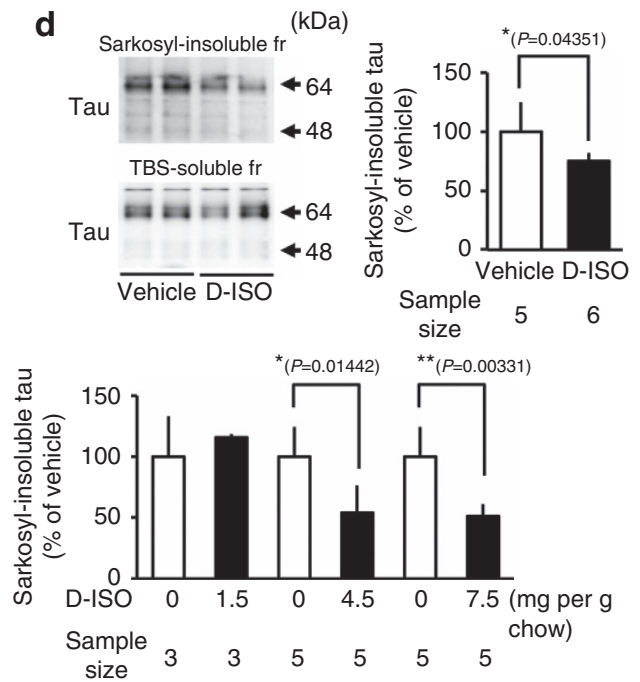

g Temporal area

h Basolateral amygdala
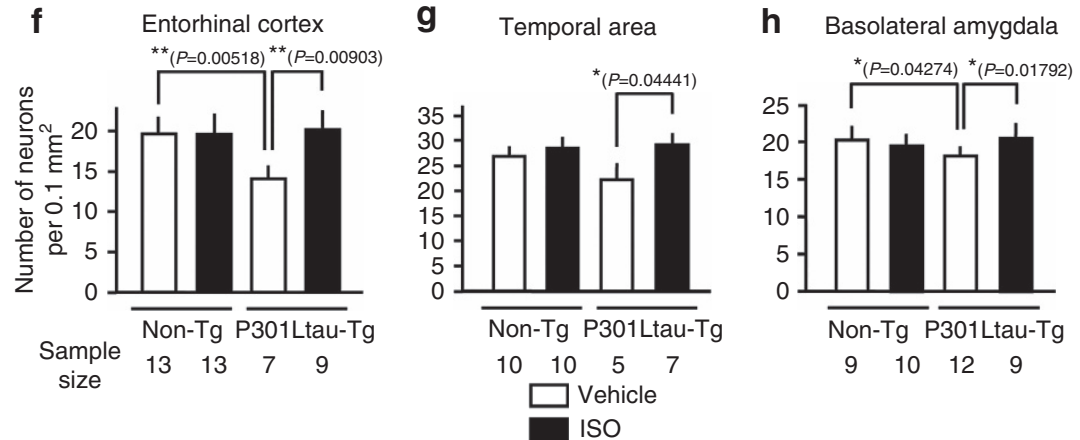

i Open field test $(0-1 \mathrm{~min})$ ${ }^{*}(P=0.01381) \quad{ }^{*}(P=0.01825)$

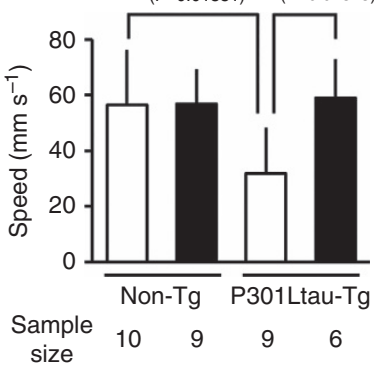

j

Open field test (0-30 $\mathrm{min})$

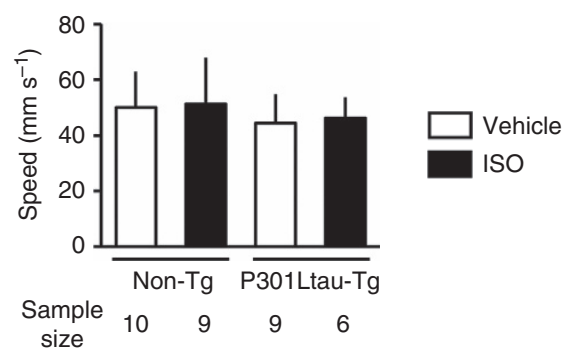

Figure 7 | Inhibition of tau aggregation and neuronal loss by isoproterenol (ISO) in vivo. Mice expressing human 2N4R tau (P301L) and non-transgenic (Tg) littermate were administrated with vehicle, $1.5 \mathrm{mg}$ ISO (a,b,f-j) and D-ISO (c,d) per g chow for 3 months. The mice were treated with 1.5-7.5 mg D-ISO per g chow for 2 months (e). In the cerebral cortex $(\mathbf{a}, \mathbf{c})$ and hippocampus $(\mathbf{b}, \mathbf{d}, \mathbf{e})$, levels of Sarkosyl-insoluble and TBS-soluble tau were analysed by western blot and quantified. JM antibody that recognized total tau was used for detection. Densitometry of tau immunoreactivity was quantified and intensity levels of Sarkosyl-insoluble tau normalized to that of TBS-soluble tau. Results are shown as percentage of control (mean \pm s.d. of 3-12 samples from 3-8 mice from three repeated independent experiments). ${ }^{\star} P<0.05 ;{ }^{\star \star} P<0.01$ (unpaired Welch's (a) and Student's t-tests (b-e)). Coronal sections (4- $\left.\mu \mathrm{m}\right)$ of mice administrated the vehicle and ISO for 3 months were stained with cresyl violet. The number of neurons in entorhinal cortex (f), temporal area (g) and basolateral amygdala (h) were counted under a microscope assisted with the Neurolucida tracing system. Results are shown as mean \pm s.d. of 5-13 samples from 3-5 mice from two independent experiments. ${ }^{\star} P<0.05$; ${ }^{\star \star} P<0.01$ (one-way analysis of variance (ANOVA), with Tukey's multiple comparisons test). Locomotor activity in ISO-treated and untreated mice was monitored in an open field arena $\left(50 \times 50 \times 40 \mathrm{~cm}^{3}\right)$ as described in the Methods. Velocity over the 1st minute (i) or 30th minute of testing (j) was determined by computing the time intervals between sequential positions of the mice in each video frame. Results are shown as mean \pm s.d. of 6-10 mice. ${ }^{\star} P<0.05$ (one-way ANOVA, with Tukey's multiple comparisons test). Sample sizes in each experiment are presented in each figure, and mice numbers are described in Supplementary Table 2. fr, fraction. 
Preparation of recombinant tau protein. Various human tau cDNA was constructed in a pRK172 vector based on the longest form of human wild-type tau encoded 441 amino acid (2N4R); deletion of amino acids at positions 252 to 376 ( $\triangle \mathrm{MTBR})$, positions 306 to $311(\Delta \mathrm{PHF} 6)$, positions 275 to $280\left(\Delta \mathrm{PHF}^{*}\right)$ and with substitutions at C291A and C322A (C291, 322A). A construct that encoded 2N3R tau with a mutation at C322A (2N3R-C322A) was produced. Each recombinant tau was expressed in Escherichia coli BL21 (DE3) and purified by modified method reported previously ${ }^{33}$. After $E$. coli expressing tau was sonicated and boiled, recombinant tau proteins in the heat-stable fraction was purified by ion-exchange chromatography (P11; GE Healthcare, or Cellufine Phosphate; JNC Corp.), ammonium sulfate fractionation, gel filtration chromatography (NAP10 column; GE Healthcare) and reverse phase-HPLC (COSMOSIL Protein-R Waters; Nacalai Tesque Inc.). After freeze-drying, recombinant tau proteins were dissolved in milliQ water and stored at $-80^{\circ} \mathrm{C}$ as a stock solution.

ThT assay. ThT binding was measured with modified method reported previously $^{33}$. Recombinant wild-type $2 \mathrm{~N} 4 \mathrm{R}$ tau $(10 \mu \mathrm{M})$, compounds (indicated concentration) and ThT $(10 \mu \mathrm{M})$ were mixed in the HEPES buffer $(10 \mathrm{mM}$ HEPES, $\mathrm{pH}=7.4 ; 100 \mathrm{mM} \mathrm{NaCl})$, and incubated with heparin $\left(0.06 \mathrm{mg} \mathrm{ml}^{-1}\right.$; Acros Organics) at $37^{\circ} \mathrm{C}$. At specific time points, fluorescence generated by the binding of ThT to tau aggregates was measured (excitation wavelength: $444 \mathrm{~nm}$; emission wavelength: $485 \mathrm{~nm}$ ). The tau aggregation mixture was collected $120 \mathrm{~h}$ after incubation, and analysed with a pelleting assay, sucrose density gradient centrifugation or AFM.

Sucrose density gradient centrifugation. Sucrose density gradient centrifugation was performed as described previously ${ }^{33}$. Tau aggregation mixture $(1 \mathrm{ml})$ was layered on top of sucrose step gradients (each $1 \mathrm{ml}$ of 10, 20, 30, 40 and $50 \%$ sucrose in HEPES buffer ( $\mathrm{pH}=7.4)$ ) was centrifuged $(50,000$ r.p.m., $2 \mathrm{~h}$ ) in a MLS50 rotor (Beckman Coulter) and separated into fractions. Pellet (Pel; Fraction 6) was suspended in $1 \mathrm{ml}$ of buffer containing HEPES buffer, and the recovered tau in each fraction was evaluated with western blotting.

Pelleting assay. Pelleting assay was performed by modified method reported previously ${ }^{54}$. After ThT assay, samples including tau aggregates were centrifuged (70,000 r.p.m., 2 h) in TLA100.4 or TLA110 rotors (Beckman Coulter) and separated into supernatant and pellet. After the pellet was suspended and sonicated in Laemmli SB including 2-mercaptoethanol, they were boiled for $5 \mathrm{~min}$.

Cell culture. Neuro2A cell line stably expressing myc-tagged tau (P301L) was established according to a previously described method ${ }^{38}$. Cells were cultured in Dulbecco's Modified Eagle's medium supplemented with $10 \%$ fetal bovine serum, $50 \mu \mathrm{g} \mathrm{ml}^{-1}$ gentamicin, $5 \mu \mathrm{g} \mathrm{ml}^{-1}$ puromycin and $1 \mathrm{mg} \mathrm{ml}^{-1} \mathrm{G} 418$ at $37^{\circ} \mathrm{C}$ under $5 \% \mathrm{CO}_{2}$. Cells at $80-90 \%$ confluency were plated in mediums without puromycin and G418. Cells were treated with ISO $(0.01,0.1$ and $1 \mu \mathrm{M})$, lithium chloride $(10 \mathrm{mM})$ as a positive control that is a glycogen synthase kinase inhibitor, or sodium chloride $(10 \mathrm{mM})$ as a negative control. To confirm the relationship between tau aggregation with $\beta$ adrenergic effect by ISO, cells were treated with $1 \mu \mathrm{M}$ ISO, $30 \mathrm{~min}$ after pretreating with or without 1 or $10 \mu \mathrm{M}$ propranolol, which is a competitive adrenergic $\beta$ blocker. Cells were sonicated in modified RIPA buffer containing $50 \mathrm{mM}$ Tris $(\mathrm{pH}=7.4), 1 \% \mathrm{NP}-40,0.25 \%$ sodium deoxycholate, $150 \mathrm{mM} \mathrm{NaCl}, 1 \mathrm{mM}$ ethylene glycol tetraacetic acid (EGTA), protease inhibitors $\left(5 \mu \mathrm{g} \mathrm{ml}^{-1}\right.$ pepstatin, $5 \mu \mathrm{g} \mathrm{ml}^{-1}$ leupeptin, $2 \mu \mathrm{g} \mathrm{ml}^{-1}$ aprotinin and $0.5 \mathrm{mM} 4$-(2-aminoethyl)benzenesulfonyl fluoride hydrochloride), and phosphatase inhibitors ( $1 \mathrm{mM}$ okadaic acid, $1 \mathrm{mM} \mathrm{Na}_{3} \mathrm{VO}_{4}$ and $1 \mathrm{mM} \mathrm{NaF}$ ) $48 \mathrm{~h}$ after treatment. The lysates $(1 \mathrm{ml})$ were layered onto $0.32 \mathrm{M}$ sucrose containing $10 \mathrm{mM}$ Tris $(\mathrm{pH}=7.4), 0.8 \mathrm{M} \mathrm{NaCl}$ and $1 \mathrm{mM}$ EGTA, and centrifuged (15,000 r.p.m., $10 \mathrm{~min}, 4^{\circ} \mathrm{C}$ ) in an ARO15-24 rotor (Tomy Seiko). The upper part ( $1 \mathrm{ml}$ ) was transferred to $1.5-\mathrm{ml}$ tubes (357448; Beckman Coulter), centrifuged $\left(50,000\right.$ r.p.m., $\left.30 \mathrm{~min}, 4^{\circ} \mathrm{C}\right)$ in a TLA55 rotor (Beckman Coulter) and separated into supernatant (RIPA-soluble fraction) and pellet. After the pellets were sonicated in $1 \%$ SDS/RIPA and $1 \%$ SDS/TBS buffers, they were centrifuged (50,000 r.p.m., $1 \mathrm{~h}, 4^{\circ} \mathrm{C}$ ) in a TLA55 rotor and separated into supernatant and pellet. Pel (SDS-insoluble fraction) was dissolved in $70 \%$ formic acid and air-dried. Samples from RIPA-soluble and SDS-insoluble fractions were dissolved in Laemmli SB including 2-mercaptoethanol, and then boiled for $5 \mathrm{~min}$.

AFM observation. Morphology of the recombinant tau aggregate was observed under $\mathrm{AFM}^{33}$. After ThT assay, samples including tau aggregates were loaded to mica and incubated at room temperature for $30 \mathrm{~min}$ in a moist box. The mica was washed with milliQ water and then cantilever (OMCL-TR400PSA; Olympus) detected tau aggregate with 3D-Stand Alone AFM (Asylum Research) under the tapping mode. Major and minor axis of tau aggregates and the number of tau aggregates were determined by image analysis using Matlab-based software (MathWorks Co. Ltd.).
Tau oligomerization assay. After recombinant wild-type $2 \mathrm{~N} 4 \mathrm{R}$ tau protein $(10 \mu \mathrm{M})$, and ISO $(100 \mu \mathrm{M})$ or methylene blue $(100 \mu \mathrm{M})$ mixing with buffer containing HEPES $(10 \mathrm{mM}, \mathrm{pH}=7.4)$ and $\mathrm{NaCl}(100 \mathrm{mM})$, heparin sulfate $\left(0.06 \mathrm{mg} \mathrm{ml}^{-1}\right)$ was added, and incubated at $37^{\circ} \mathrm{C}$. In each time point $10-60 \mathrm{~min}$, tau aggregation mixture were collected and dissolved in Laemmli SB without 2-mercaptoethanol. Tau protein in the samples was separated in SDS-polyacrylamide gel electrophoresis gel without dithiothreitol buffer and visualized in western blotting (Figs $4 \mathrm{c}$ and 5).

Binding assay with FG beads. To determine binding of tau and ISO, we prepared Streptavidin FG beads ( $5 \mathrm{mg} \mathrm{ml}^{-1}$; Tamagawa Seiki) reacted with biotinylated ISO $\left(62.5 \mu \mathrm{M}\right.$; Supplementary Fig. 1A), and Streptavidin FG beads $\left(5 \mathrm{mg} \mathrm{ml}^{-1}\right)$ reacted with biotin $(62.5 \mu \mathrm{M})$ as a negative control. Avidin-biotin complexes were formed by incubation for $1 \mathrm{~h}$ at $4^{\circ} \mathrm{C}$. After recombinant wild-type $2 \mathrm{~N} 4 \mathrm{R}$ tau $(0.6 \mu \mathrm{M}$; input) was reacted with the complexes by rotation $\left(4 \mathrm{~h}\right.$ at $\left.4^{\circ} \mathrm{C}\right)$, they were centrifuged and separated into pellet and supernatant. After washing the pellet with $100 \mathrm{mM} \mathrm{KCl}$ buffer ( $\mathrm{pH}$ 7.4) thrice, the pellet was suspended and incubated in $1 \mathrm{M}$ $\mathrm{KCl}$ solution for $5 \mathrm{~min}$ on ice. The suspension was centrifuged, the supernatant $(\mathrm{KCl})$ was analysed, or the pellet was suspended in Laemmli SB without 2-mercaptoethanol, incubated for $15 \mathrm{~min}$ at $60^{\circ} \mathrm{C}$ and separated into Pel and supernatant (SB; Supplementary Fig. 1b). The Input, $\mathrm{KCl}$ and Pel were solubilized or resuspended in Laemmli SB. Tau protein in the samples was detected with western blotting. To determine binding region, R1 (tapvpmpdlknvkskigstenlkhqpgggk), R2 (vqiinkkldlsnvqskcgskdnikhvpgggs), R3 (vqivykpvdlskvtskcgslgnihhkpgggq) or R4 (vevksekldfkdrvqskigsldnithvpgggn) peptides $(120 \mu \mathrm{M})$ were pretreated with the avidin-biotin complexes before incubation with recombinant wild-type $2 \mathrm{~N} 4 \mathrm{R}$ tau.

MASS spectrometry. Recombinant R3' (skvtskcgslgn) and mutant Cys $\rightarrow$ Ala R3 (skvtskagslgn) peptides $(10 \mu \mathrm{M})$ were incubated with compounds $(100 \mu \mathrm{M})$ for 5 days at $37^{\circ} \mathrm{C}$. The samples were diluted 20 times with milliQ water and loaded to spot under presence of $\alpha$-Cyano-4-hydroxycinnamic acid $\left(10 \mathrm{mg} \mathrm{ml}^{-1}\right)$. After drying, the samples were analysed using 4800 plus MALDI Tof/Tof analyzer (Applied Biosystems).

Animals. Transgenic mice expressing 2N4R tau (P301L) with myc- and flag-tag at $\mathrm{N}$ - and C-terminal under CaM kinase II promoter was created previously ${ }^{26}$. Male P301L tau-transgenic mice 17 - to 18 -month old were treated with ISO or D-ISO (1.5-7.5 $\mathrm{mg} \mathrm{g}^{-1}$ chow) for 2 or 3 months. The numbers of animals used in each experiment are indicating in Supplementary Table 2. All experimental procedures used in this study were approved by the Committee of Animal Experiments at the National Center for Geriatrics and Gerontology.

Tissue extraction. P301L tau-transgenic mice were anaesthetized and killed after treatment with the compounds; the hippocampus and cerebral cortex were collected. The tissues were homogenized in TBS buffer containing $50 \mathrm{mM}$ Tris $(\mathrm{pH}=7.4), 150 \mathrm{mM} \mathrm{NaCl}, 1 \mathrm{mM}$ EGTA, $1 \mathrm{mM}$ EDTA, protease inhibitors and phosphatase inhibitors. The homogenates were centrifuged (23,000 r.p.m., $15 \mathrm{~min}$, $4^{\circ} \mathrm{C}$ ) in a TLA55 rotor and separated into supernatant (TBS-soluble fraction) and pellet. Pellets were resuspended in $0.32 \mathrm{M}$ sucrose containing $10 \mathrm{mM}$ Tris ( $\mathrm{pH} 7.4)$, $0.8 \mathrm{M} \mathrm{NaCl}$ and $1 \mathrm{mM}$ EGTA and centrifuged $\left(23,000\right.$ r.p.m., $15 \mathrm{~min}, 4^{\circ} \mathrm{C}$ ) in a TLA55 rotor. Supernatants were collected and treated with $1 \%$ Sarkosyl for $1 \mathrm{~h}$ at $37^{\circ} \mathrm{C}$. They were centrifuged $\left(60,000\right.$ r.p.m., $\left.1 \mathrm{~h}, 4^{\circ} \mathrm{C}\right)$ in a TLA 100.4 or 110 rotors and separated into supernatant and pellet (Sarkosyl-insoluble fraction). Samples from TBS-soluble and Sarkosyl-insoluble fractions were dissolved in Laemmli SB including 2-mercaptoethanol, and then boiled for $5 \mathrm{~min}$.

Count of neuronal cells. Number of neuronal cells in the brain was measured with modified method reported previously ${ }^{26}$. After P301L tau-transgenic mice were anaesthetized and transcardially perfused with $10 \%$ formalin, brains were fixed in $10 \%$ formalin solutions for $48 \mathrm{~h}$. Coronal sections $(4 \mu \mathrm{m})$ were produced from brains embedded by paraffin, and stained with cresyl violet. We counted the number of neuronal cells in the sections using microscope linked to a Neurolucida tracing system (MicroBrightField Inc.).

Western blotting. Samples dissolved in Laemmli SB were separated by Novex 3-8\% Tris-Acetate Gel (binding assay and tau oligomerization assay; Invitrogen) or SuperSep Ace 5-20\% gel (other assays; WAKO Pure Chemical Industries) and transferred onto membranes with semi-dry transfer systems (Bio-Rad Laboratory). The membrane was blocked with $5 \%$ milk in PBS-T for $1 \mathrm{~h}$ at room temperature. They were probed with antibodies overnight at $4{ }^{\circ} \mathrm{C}$. After washing the membrane with PBS-T, blots were incubated with horseradish peroxidase-linked second antibodies and then examined by enhanced chemiluminescence detection on Las3000 or Las4000 (GE Healthcare).

Open field test. Mice were placed in the centre of an open field apparatus $\left(50 \times 50 \times 40 \mathrm{~cm}^{3}\right.$; O'Hara Co., Ltd.), and their locomotor activity was monitored with a CCD camera; digital data of real-time images were recorded using the public 
domain NIH Image J software (http://rsb.info.nih.gov/nih-image/). Images were sampled at $2 \mathrm{~Hz}$. Data were analysed using customized Matlab-based software, using an image analysis tool box (Mathworks Co. Ltd.). During testing, the sequential position of the mouse was determined in each video frame from which locomotor speed was calculated.

Mn-enhanced magnetic resonance imaging. Mn-enhanced MRI was performed as previously described ${ }^{55}$. Mice were given an intraperitoneal injection of $30 \mathrm{mM}$ $\mathrm{MnCl}_{2}\left(100 \mu \mathrm{mol} \mathrm{kg}^{-1}\right)$ and returned to their home cages. After $1 \mathrm{~h}$, mice were exposed successively to three different novel places separated by a small (diameter, $30 \mathrm{~cm}$; height, $30 \mathrm{~cm}$ ) and big (diameter, $60 \mathrm{~cm}$; height, $30 \mathrm{~cm}$ ) transparent plastic wall and was allowed to explore for $120 \mathrm{~min}$. Thereafter, mice were returned to their home cages for $1 \mathrm{~h}$ before being anaesthetized with isoflurane $(0.5-1.5 \%$ in air) and placement in a Bruker 3T MR scanner; during scanning, breathing and depth of anaesthesia was continuously monitored, with breathing rate maintained at $80-100$ breaths per min. Mn-enhanced MRI images were visualized with open-source Osirix software (version 2.5) that allowed navigation through multidimensional DICOM images; relative regional brain activity was determined by measuring MR signal intensities, normalized to the mean signal intensity in the dorsal striatum.

Measurement of ISO levels in the blood and brain. Male C57BL/6J mice $(n=7)$ were administered ISO ( $1.5 \mathrm{mg} \mathrm{g}^{-1}$ chow) for 2 weeks before sacrifice when blood plasma and whole brain were collected. The samples were deproteinized using acetonitrile before determination of ISO concentrations using an LC-MS instrument (UPLC/Quattro Premier XE; Waters).

Statistical analysis. Data are expressed as means \pm s.d. The significance of differences between two groups was assessed by Student's or Welch's $t$-tests, and differences between multiple groups were assessed by one-way analysis of variance and Tukey's multiple comparisons test, using PRISM4 (GraphPad Software Inc.). $P<0.05$ was considered statistically significant.

\section{References}

1. Schirmer, R. H., Adler, H., Pickhardt, M. \& Mandelkow, E. "Lest we forget you--methylene blue...". Neurobiol. Aging 32, 2325.e2327-2316 (2011).

2. Selkoe, D. J. Alzheimer disease: mechanistic understanding predicts novel therapies. Ann. Intern. Med. 140, 627-638 (2004).

3. Desai, A. K. \& Chand, P. Tau-based therapies for Alzheimer's disease: wave of the future. Primary Psychiatry 16, 40-46 (2009)

4. Hardy, J. \& Selkoe, D. J. The amyloid hypothesis of Alzheimer's disease: progress and problems on the road to therapeutics. Science 297, 353-356 (2002).

5. Kosik, K. S. Traveling the tau pathway: a personal account. J. Alzheimers Dis. 9, 251-256 (2006).

6. Liu, J. et al. Amyloid-beta induces caspase-dependent loss of PSD-95 and synaptophysin through NMDA receptors. J. Alzheimers Dis. 22, 541-556 (2010).

7. Roselli, F. et al. Soluble beta-amyloid1-40 induces NMDA-dependent degradation of postsynaptic density-95 at glutamatergic synapses. J. Neurosci. 25, 11061-11070 (2005).

8. Ittner, L. M. et al. Dendritic function of tau mediates amyloid-beta toxicity in Alzheimer's disease mouse models. Cell 142, 387-397 (2010).

9. Roberson, E. D. et al. Reducing endogenous tau ameliorates amyloid betainduced deficits in an Alzheimer's disease mouse model. Science 316, 750-754 (2007).

10. Holmes, C. et al. Long-term effects of Abeta42 immunisation in Alzheimer's disease: follow-up of a randomised, placebo-controlled phase I trial. Lancet 372, 216-223 (2008).

11. Rosenblum, W. I. Why Alzheimer trials fail: removing soluble oligomeric beta amyloid is essential, inconsistent, and difficult. Neurobiol. Aging 35, 969-974 (2014).

12. Giacobini, E. \& Gold, G. Alzheimer disease therapy--moving from amyloid-beta to tau. Nat. Rev. Neurol. 9, 677-686 (2013).

13. Guillozet, A. L., Weintraub, S., Mash, D. C. \& Mesulam, M. M. Neurofibrillary tangles, amyloid, and memory in aging and mild cognitive impairment. Arch. Neurol. 60, 729-736 (2003).

14. Gomez-Isla, T. et al. Clinical and pathological correlates of apolipoprotein $\mathrm{E}$ epsilon 4 in Alzheimer's disease. Ann. Neurol. 39, 62-70 (1996).

15. Bondareff, W., Mountjoy, C. Q., Roth, M. \& Hauser, D. L. Neurofibrillary degeneration and neuronal loss in Alzheimer's disease. Neurobiol. Aging 10, 709-715 (1989).

16. Bobinski, M. et al. Neurofibrillary pathology--correlation with hippocampal formation atrophy in Alzheimer disease. Neurobiol. Aging 17, 909-919 (1996).

17. Hutton, M. et al. Association of missense and 5'-splice-site mutations in tau with the inherited dementia FTDP-17. Nature 393, 702-705 (1998).
18. Poorkaj, P. et al. Tau is a candidate gene for chromosome 17 frontotemporal dementia. Ann. Neurol. 43, 815-825 (1998).

19. Spillantini, M. G. et al. Mutation in the tau gene in familial multiple system tauopathy with presenile dementia. Proc. Natl Acad. Sci. USA 95, 7737-7741 (1998).

20. Yoshiyama, Y. et al. Synapse loss and microglial activation precede tangles in a P301S tauopathy mouse model. Neuron 53, 337-351 (2007).

21. Allen, B. et al. Abundant tau filaments and nonapoptotic neurodegeneration in transgenic mice expressing human P301S tau protein. J. Neurosci. 22, 9340-9351 (2002).

22. Lewis, J. et al. Neurofibrillary tangles, amyotrophy and progressive motor disturbance in mice expressing mutant (P301L) tau protein. Nat. Genet. 25, 402-405 (2000).

23. Tanemura, K. et al. Neurodegeneration with tau accumulation in a transgenic mouse expressing V337M human tau. J. Neurosci. 22, 133-141 (2002).

24. Tatebayashi, Y. et al. Tau filament formation and associative memory deficit in aged mice expressing mutant (R406W) human tau. Proc. Natl Acad. Sci. USA 99, 13896-13901 (2002).

25. Santacruz, K. et al. Tau suppression in a neurodegenerative mouse model improves memory function. Science 309, 476-481 (2005).

26. Kimura, T. et al. Aggregation of detergent-insoluble tau is involved in neuronal loss but not in synaptic loss. J. Biol. Chem. 285, 38692-38699 (2010).

27. Cowan, C. M. et al. Modelling tauopathies in Drosophila: insights from the fruit fly. Int. J. Alzheimers Dis. 2011, 598157 (2011).

28. Bretteville, A. et al. Hypothermia-induced hyperphosphorylation: a new model to study tau kinase inhibitors. Sci. Rep. 2, 480 (2012).

29. Gong, C. X. et al. Phosphorylation of microtubule-associated protein tau is regulated by protein phosphatase $2 \mathrm{~A}$ in mammalian brain. Implications for neurofibrillary degeneration in Alzheimer's disease. J. Biol. Chem. 275, 5535-5544 (2000).

30. Schneider, A., Biernat, J., von Bergen, M., Mandelkow, E. \& Mandelkow, E. M Phosphorylation that detaches tau protein from microtubules (Ser262, Ser214) also protects it against aggregation into Alzheimer paired helical filaments. Biochemistry 38, 3549-3558 (1999).

31. Wang, Y. P., Biernat, J., Pickhardt, M., Mandelkow, E. \& Mandelkow, E. M. Stepwise proteolysis liberates tau fragments that nucleate the Alzheimer-like aggregation of full-length tau in a neuronal cell model. Proc. Natl Acad. Sci. USA 104, 10252-10257 (2007).

32. Sahara, N. et al. Assembly of two distinct dimers and higher-order oligomers from full-length tau. Eur. J. Neurosci. 25, 3020-3029 (2007).

33. Maeda, S. et al. Granular tau oligomers as intermediates of tau filaments. Biochemistry 46, 3856-3861 (2007).

34. Maeda, S. et al. Increased levels of granular tau oligomers: an early sign of brain aging and Alzheimer's disease. Neurosci. Res. 54, 197-201 (2006).

35. Takashima, A. Tauopathies and tau oligomers. J. Alzheimers Dis. 37, 565-568 (2013).

36. Chi, O. Z., Wang, G., Chang, Q. \& Weiss, H. R. Effects of isoproterenol on blood-brain barrier permeability in rats. Neurol. Res. 20, 259-264 (1998).

37. Eriksson, T. \& Carlsson, A. Isoprenaline increases brain concentrations of administered L-dopa and L-tryptophan in the rat. Psychopharmacology (Berl) 77, 98-100 (1982).

38. Hatakeyama, S. et al. U-box protein carboxyl terminus of Hsc70-interacting protein (CHIP) mediates poly-ubiquitylation preferentially on four-repeat Tau and is involved in neurodegeneration of tauopathy. J. Neurochem. 91, 299-307 (2004).

39. Bhattacharya, K., Rank, K. B., Evans, D. B. \& Sharma, S. K. Role of cysteine-291 and cysteine-322 in the polymerization of human tau into Alzheimer-like filaments. Biochem. Biophys. Res. Commun. 285, 20-26 (2001).

40. von Bergen, M. et al. Assembly of tau protein into Alzheimer paired helical filaments depends on a local sequence motif ((306)VQIVYK $(311))$ forming beta structure. Proc. Natl Acad. Sci. USA 97, 5129-5134 (2000).

41. von Bergen, M. et al. Mutations of tau protein in frontotemporal dementia promote aggregation of paired helical filaments by enhancing local betastructure. J. Biol. Chem. 276, 48165-48174 (2001).

42. Jagoe, C. T., Kreifels, S. E. \& Li, J. Covalent binding of catechols to Src family SH2 domains. Bioorganic Med. Chem. Lett. 7, 113-116 (1997).

43. Lipton, S. A. Pathologically activated therapeutics for neuroprotection. Nat. Rev. Neurosci. 8, 803-808 (2007).

44. Guin, P. S., Das, S. \& Mandal, P. Electrochemical reduction of quinones in different media: a review. Int. J. Electrochem. 2011, 816202 (2011).

45. Nakashima, H. et al. Effects of alpha-tocopherol on an animal model of tauopathies. Free Radic. Biol. Med. 37, 176-186 (2004).

46. Dias-Santagata, D., Fulga, T. A., Duttaroy, A. \& Feany, M. B. Oxidative stress mediates tau-induced neurodegeneration in Drosophila. J. Clin. Invest. 117, 236-245 (2007)

47. Gruning, C. S. et al. Alternative conformations of the Tau repeat domain in complex with an engineered binding protein. J. Biol. Chem. 289, 23209-23218 (2014). 
48. Conolly, M. E. et al. Metabolism of isoprenaline in dog and man. Br. J. Pharmac. 46, 458-472 (1972).

49. Singh, J. et al. Structure-based design of a potent, selective, and irreversible inhibitor of the catalytic domain of the erbB receptor subfamily of protein tyrosine kinases. J. Med. Chem. 40, 1130-1135 (1997).

50. Takashima, A. et al. Presenilin 1 associates with glycogen synthase kinase-3beta and its substrate tau. Proc. Natl Acad. Sci. USA 95, 9637-9641 (1998).

51. Hagiwara, K. et al. Discovery of novel antiviral agents directed against the influenza A virus nucleoprotein using photo-cross-linked chemical arrays. Biochem. Biophys. Res. Commun. 394, 721-727 (2010).

52. Kanoh, N. et al. Photo-cross-linked small-molecule microarrays as chemical genomic tools for dissecting protein-ligand interactions. Chem. Asian J. 1, 789-797 (2006).

53. Miyazaki, I., Simizu, S., Ichimiya, H., Kawatani, M. \& Osada, H. Robust and systematic drug screening method using chemical arrays and the protein library: identification of novel inhibitors of carbonic anhydrase II. Biosci. Biotechnol. Biochem. 72, 2739-2749 (2008).

54. Pickhardt, M. et al. Screening for inhibitors of tau polymerization. Curr. Alzheimer Res. 2, 219-226 (2005).

55. Kimura, T. et al. Hyperphosphorylated tau in parahippocampal cortex impairs place learning in aged mice expressing wild-type human tau. $E M B O J . \mathbf{2 6}$, 5143-5152 (2007).

\section{Acknowledgements}

We thank Drs Tamio Saito and Aya Asami (RIKEN, Wako, Japan) for kindly providing 6,788 test compounds; Dr Toshihide Hashimoto (Eisai Co., Ltd., Tukuba, Japan) for kindly providing biotinylated ISO; Ms. Miyuki Murayama (RIKEN, Wako, Japan) and Mr Shunji Yamashita (O’HARA \& Co., Ltd., Tokyo Japan) for valuable technical advice; and Ms Midori Yamamoto (National Center for Geriatrics and Gerontology, Obu, Japan) and Mr Tatsuya Mizoroki (Institute of Immunology Co., Ltd., Utsunomiya, Japan) for assistance in the maintenance of mice.

This work was supported by JSPS KAKENHI Grant Number 23790313 (to Y.S.), Mext Grant-in-aid project, Scientific Research on Innovation Area (Brain Protein Aging and Dementia control (to A.T.), Brain environment (to A.T.)) and Strategic Research
Program for Brain Science ('Integrated Research on Neuropsychiatric Disorders') from Japan Agency for Medical Research and development, AMED (to Y.I., A.T. and H.S.) and Intramural grant of NCGG (to A.T.).

\section{Author contributions}

M.Y. and A.Su. performed in vivo experiments. O.F.X.A. provided critical suggestions during writing of the manuscript. S.M., H.O., Y.K. and A.Sa. performed the chemical array screening and evaluated the data. T.M. and Y.I. performed MS analysis. T.K. calculated major axis and number of tau aggregates in images obtained by AFM using Matlab. M.S. and H.K. analysed chemical data. H.S. synthesized and purified D-ISO. Y.Y provided critical techniques for ThT assay and cultured cell analysis. Y.S. and A.T. designed the experiment, and evaluated all data. Y.S. and A.T. wrote the manuscript. A.T. supervised the study.

\section{Additional information}

Supplementary Information accompanies this paper at http://www.nature.com/ naturecommunications

Competing financial interests: The authors declare no competing financial interests.

Reprints and permission information is available online at http://npg.nature.com/ reprintsandpermissions/

How to cite this article: Soeda, Y. et al. Toxic tau oligomer formation blocked by capping of cysteine residues with 1,2-dihydroxybenzene groups. Nat. Commun. 6:10216 doi: $10.1038 /$ ncomms10216 (2015).

\section{(c) (i)}

This work is licensed under a Creative Commons Attribution 4.0 International License. The images or other third party material in this article are included in the article's Creative Commons license, unless indicated otherwise in the credit line; if the material is not included under the Creative Commons license, users will need to obtain permission from the license holder to reproduce the material. To view a copy of this license, visit http://creativecommons.org/licenses/by/4.0/ 